\title{
əThe Role of Curvature in Modifying Frontal Instabilities. Part II: Application of the Criterion to Curved Density Fronts at Low Richardson Numbers 0
}

\author{
Christian E. Buckingham, ${ }^{\mathrm{a}, \mathrm{b}}$ JONATHAN Gula, ${ }^{\mathrm{a}}$ AND XAVIER CARTON ${ }^{\mathrm{a}}$ \\ ${ }^{a}$ Université de Bretagne Occidentale, CNRS, IRD, Ifremer, Laboratoire d'Océanographie Physique et Spatiale, IUEM, \\ Plouzané, France \\ ${ }^{\mathrm{b}}$ British Antarctic Survey, Cambridge, United Kingdom
}

(Manuscript received 4 November 2019, in final form 23 October 2020)

\begin{abstract}
We continue our study of the role of curvature in modifying frontal stability. In Part I, we obtained an instability criterion valid for curved fronts and vortices in gradient wind balance (GWB): $\Phi^{\prime}=L^{\prime} q^{\prime}<0$, where $L^{\prime}$ and $q^{\prime}$ are the nondimensional absolute angular momentum and Ertel potential vorticity (PV), respectively. In Part II, we investigate this criterion in a parameter space representative of low-Richardson-number fronts and vortices in GWB. An interesting outcome is that, for Richardson numbers near 1, anticyclonic flows increase in $q^{\prime}$, while cyclonic flows decrease in $q^{\prime}$, tending to stabilize anticyclonic and destabilize cyclonic flow. Although stability is marginal or weak for anticyclonic flow (owing to multiplication by $L^{\prime}$ ), the destabilization of cyclonic flow is pronounced, and may help to explain an observed asymmetry in the distribution of small-scale, coherent vortices in the ocean interior. We are referring to midlatitude submesoscale and polar mesoscale vortices that are generated by friction and/or buoyancy forcing within boundary layers but that are often documented outside these layers. A comparison is made between several documented vortices and predicted stability maps, providing support for the proposed mechanism. A simple expression, which is a root of the stability discriminant $\Phi^{\prime}$, explains the observed asymmetry in the distribution of vorticity. In conclusion, the generalized criterion is consistent with theory, observations, and recent modeling studies and demonstrates that curvature in low-stratified environments can destabilize cyclonic and stabilize anticyclonic fronts and vortices to symmetric instability. The results may have implications for Earth system models.
\end{abstract}

SIGNIFICANCE STATEMENT: Considerable progress has been made by considering ocean fronts to be in geostrophic balance. By this, we mean that fluid parcels accelerate as a result of horizontal pressure gradients and Earth's rotation. A good example of this is in our efforts to understand symmetric instability, a process thought to impact energy, buoyancy, and tracer budgets in the ocean. However, we wanted to know how the physics might change if we accounted for centrifugal forces, or curvature. It turns out that this same question had been asked and answered nearly 100 years ago. However, the new criteria that we introduce in Part I yield (in Part II) one result that is new: in low-stratified waters, curved cyclonic fronts become strongly unstable and curved anticyclonic fronts become marginally stable. This suggests that highly curved cyclonic fronts and vortices are symmetrically unstable, with potential implications for the aforementioned budgets.

KEYWORDS: Eddies; Fronts; Instability; Ocean circulation; Potential vorticity; Frontogenesis/frontolysis; Vortices; Angular momentum

\section{Introduction}

Symmetric instability is a vertical shear instability found within baroclinic fronts. A unique feature of symmetric instability is that the flow can be both statically stable (i.e., gravitationally stable) and inertially or centrifugally stable, and yet is still "symmetrically unstable" owing to the baroclinic nature of the flow. The instability is often triggered by internal waves incident on the front (Mooers 1975; Li et al. 2019), resulting in parcel motion approximately along isopycnals and inclined

๑ Denotes content that is immediately available upon publication as open access.

Supplemental information related to this paper is available at the Journals Online website: https://doi.org/10.1175/JPO-D-20-0258.s1.

Corresponding author: Christian E. Buckingham, christian. buckingham@univ-brest.fr relative to the horizontal. This slanted parcel motion is why the instability has also been referred to as slant-wise convection (Thorpe and Rotunno 1989), although it should be emphasized that it is associated with considerably less buoyancy flux than is typically found in gravitational instability or upright convection (Bachman et al. 2017). Also, although it occurs in baroclinic fronts and can be considered as a form of baroclinic instability (Stone 1966, 1970), the underlying dynamics and associated parcel motions are different than those found within classical baroclinic instability (Eady 1949; Charney 1947).

The subject of symmetric instability has received considerable attention recently within the context of oceanic submesoscale processes and, specifically, within the context of wind- and buoyancy-forced symmetric instability (Thomas et al. 2008; D'Asaro et al. 2011; Thomas et al. 2013; Arobone and Sarkar 2015; McWilliams 2016; Bachman et al. 2017; Skyllingstad et al. 2017; Buckingham et al. 2019). Here, the term submesoscale is used to refer to dynamics nominally encountered at lateral scales of $0.1-10 \mathrm{~km}$ and temporal scales from hours to days. When compared with flows within the 
quasigeostrophic (QG) regime, fluid flows within the submesoscale regime are found to possess elevated gradient Rossby numbers and reduced gradient Richardson numbers (Thomas et al. 2008; McWilliams 2016), revealing that they are characterized by enhanced gradients in velocity and density and yet remain strongly influenced by Earth's rotation. Moreover, within boundary layers, vertical stratification is reduced to the extent that gradient Richardson numbers within fronts can approach 1. For example, vertical stratifications of $N / f \sim 20-100$ are common, leading to low-gradient Richardson numbers. Here, $N$ is the Brunt-Väisälä, or buoyancy, frequency and $f$ is the Coriolis parameter. Within such low-stratified environments, symmetric instability within fronts is expected (Stone 1966, 1970). Examples of such boundary layers include the ocean surface, bottom, and ice-ocean boundary layers, where winds, flow over topography, or buoyancy-forced convection act to reduce $N / f$ (Legg and McWilliams 2001; Thomas and Taylor 2010; Thomas et al. 2013; Wenegrat et al. 2018; Naveira Garabato et al. 2019).

Building on several decades of research, Hoskins (1974) demonstrated that the criterion for symmetric instability can be stated in terms of the Ertel PV. Employing the model of Ooyama [1966, their Eq. (19)], Hoskins (1974) found that in the Northern Hemisphere, symmetric instability is possible within a front in thermal wind balance (TWB) when $q<$ 0 , where

$$
q=\boldsymbol{\omega}_{a} \cdot \nabla b
$$

is the Ertel PV (Ertel 1942). In this expression, $\boldsymbol{\omega}_{a}=2 \boldsymbol{\Omega}+$ $\nabla \times \mathbf{u} \approx f \hat{\mathbf{z}}+\nabla \times \mathbf{u}$ is the absolute vorticity, $\nabla \times \mathbf{u}$ is relative vorticity, and, again, $f=2|\boldsymbol{\Omega}| \sin \theta$ is the Coriolis parameter or vertical component of planetary vorticity at latitude $\theta$. Moreover, $b=-g \rho / \rho_{o}$ is buoyancy, $g$ is acceleration due to gravity, $\rho$ is density, and $\rho_{o}$ is a reference density. Multiplying by the Coriolis parameter to eliminate the hemispheric dependence, assuming stable stratification and restricting analysis away from the equator, the instability criterion can be recast in nondimensional form:

$$
q^{\prime}=1+\mathrm{Ro}-\mathrm{Ri}^{-1}<0 .
$$

Here, $\operatorname{Ro}=\bar{\zeta} / f$ is the gradient Rossby number, $\mathrm{Ri}=N^{2} /\left|\partial_{z} \mathbf{u}\right|^{2}$ is the gradient Richardson number, $N^{2}=\partial_{z} \bar{b}$ is the vertical stratification, and $\partial_{z} \mathbf{u}$ is the vertical shear of alongfront velocity $\mathbf{u}=(0, \bar{v}, 0)$. Also, overbars denote the mean quantities. This is the classic criterion for symmetric instability of a front in TWB given in terms of nondimensional numbers (Hoskins 1974). Thus, $q^{\prime}$ serves as a discriminant for stability and instability for flows in TWB.

Most oceanographic studies examining symmetric instability focus on the Ertel PV as the relevant quantity. While this is certainly appropriate for fronts in TWB, the authors are unaware of any observational studies that have examined the conservation of absolute angular momentum in concert with the Ertel PV. However, this quantity enters the expressions for stability when centrifugal forces are present, a condition that frequently occurs in the oceans owing to the curvature of fronts and vortices. Thus, the objective of the first portion of our study (Buckingham et al. 2021, hereinafter Part I) was to 1) revisit the criterion for symmetric instability, $f q<0$ (Hoskins 1974), 2) determine when the criterion applies, and 3) establish arguments for use of a different criterion as necessary. We summarize these findings below.

\section{a. A review of the relevant criterion}

By revisiting the definition of the Ertel PV, we first found that density conservation and PV conservation were inextricably linked through Ertel's PV theorem. This reaffirmed the notion that PV is a useful measure of stability in the ocean and atmosphere (Hoskins 1974; Hoskins et al. 1985). Moreover, since PV is the inner product of absolute vorticity and density gradients, and as multiplication by $f$ ensures the expression remains valid in both hemispheres, one observes that the criterion, $f q<0$, implicitly contains information essential for describing the stability of fronts. However, by itself, this argument does not yield a sufficient criterion for instability. That is, the Ertel PV, $q$, or its nondimensional form, $q^{\prime}$, is not the appropriate stability discriminant.

We then briefly discussed the frontal models of 1) Hoskins and Bretherton (1972) and 2) Shakespeare (2016). We noted that the hyperbolic condition on the partial differential equation (PDE) governing steady, secondary circulation within the front gives Hoskins's criterion: the PDE is elliptic for $f q>0$ and hyperbolic for $f q<0$ (Holton 1992). We then derived a comparable governing equation for flows in gradient wind balance (GWB), sometimes referred to as the Sawyer-Eliassen equation, obtaining the result that the hyperbolic condition on the PDE is equivalent to the criterion first proposed by Solberg (1936) and later proved sufficient by Fjortoft (1950) and Ooyama (1966). We manipulated the expression, deriving several forms of the criterion, a few of which we highlight below. Because of the connection to statements made by Rayleigh (1917) that angular momentum must always be increasing radially outward for hydrodynamic stability, one can refer to the criterion as a generalized Rayleigh criterion, a term universally adopted by the scientific community. That said, it can equivalently be thought of as a generalized Hoskins criterion, as illustrated below [cf. Eq. (6)].

\section{1) DiMENSIONAL FORMS OF THE CRITERION}

The criterion, valid for inviscid baroclinic flow on the $f$ plane, can be written in cylindrical coordinates as follows:

$$
\Phi=\left(f+\frac{2 \bar{v}}{r}\right)(f+\bar{\zeta}) N^{2}-\left(f+\frac{2 \bar{v}}{r}\right)^{2}\left|\partial_{z} \bar{v}\right|^{2}<0
$$

where $\bar{v}$ is the mean azimuthal velocity, $r$ is the radius, $\bar{\zeta}=(1 / r) \partial_{r}(r \bar{v})$ is the vertical component of mean relative vorticity, $N^{2}=\partial_{z} \bar{b}$ is vertical stratification, and $\left|\partial_{z} \bar{v}\right|^{2}$ is the square of the vertical shear. In the case of a zonally oriented meandering front (Fig. 2 in Part I), the radius of the vortex $r$ should be replaced by a signed radius of curvature $R$ and the azimuthal velocity should be replaced by an alongfront velocity - that is, a velocity in the direction of the gradient wind shear. Because the baroclinic term in Eq. (3) is negative definite, it highlights that stability is reduced at ocean fronts owing to enhanced vertical shear. 
For reference, the mean state is presumed to be in GWB and is written as

$$
\partial_{r} \bar{b}=\left(f+\frac{2 \bar{v}}{r}\right) \partial_{z} \bar{v}
$$

where $\partial_{r} \bar{b}$ is the radial buoyancy gradient.

Two useful forms of the criterion are given below. Noting that

$$
q=(f+\bar{\zeta}) N^{2}-\left(f+\frac{2 \bar{v}}{r}\right)\left|\partial_{z} \bar{v}\right|^{2}
$$

is the Ertel PV for a flow in GWB and defining $\mathrm{Cu}=2 \bar{v} /(f r)$, one can rewrite the criterion as

$$
\Phi=(1+\mathrm{Cu}) f q<0
$$

[cf. Eq. (16) in Part I]. Here, $\mathrm{Cu}$ is a signed, nondimensional number that quantifies the curvature of the flow, which we refer to as the curvature number. ${ }^{1}$ This also makes the connection to the criterion of Hoskins (1974) more obvious.

Defining $L=r \bar{v}+f r^{2} / 2$ as the absolute angular momentum of a fluid parcel (Holton 1992), one can alternatively write the instability criterion as (Kloosterziel et al. 2007; Kloosterziel 2010)

$$
\Phi=2 L q / r^{2}<0 \quad \rightarrow \quad L q<0 .
$$

In words, the criterion states that both absolute angular momentum or the Ertel PV must be positive for stable flow. If either but not both of these quantities is negative, instability will occur. It is worth noting that the right-hand side of Eq. (7) has different units than Eq. (6). In particular, $\mathrm{Cu}$ is related to $L$ as follows: $L^{\prime}=2 L /\left(f r^{2}\right)=1+\mathrm{Cu}$, where $L^{\prime}$ is a dimensionless form of absolute angular momentum. In summary, both equations demonstrate that, in the limit of zero curvature, one recovers the criterion of Hoskins (1974) valid for fronts in TWB: $f q<0$.

\section{2) NONDIMENSIONAL FORM OF THE CRITERION}

In much the same way that the criterion, $f q<0$, can be rewritten in nondimensional form valid for symmetric instability [cf. Eq. (2)], we can also write $\Phi<0$ in nondimensional form. Assuming positive vertical stratification away from the equator, one can divide Eq. (6) by $f^{2} N^{2}>0$ to obtain

$$
\Phi^{\prime}=L^{\prime} q^{\prime}=(1+\mathrm{Cu})(1+\mathrm{Ro})-(1+\mathrm{Cu})^{2} \mathrm{Ri}^{-1}<0
$$

[cf. Eq. (17) in Part I], where again $L^{\prime}=1+\mathrm{Cu}$ is a nondimensional form of absolute angular momentum and

$$
q^{\prime}=1+\mathrm{Ro}-(1+\mathrm{Cu}) \mathrm{Ri}^{-1}=0
$$

is a nondimensional form of Ertel PV for flows in GWB. As before, $\mathrm{Ro}=\bar{\zeta} / f$ is the gradient Rossby number, $\mathrm{Ri}=N^{2} /\left|\partial_{z} \mathbf{u}\right|^{2}$ is the gradient Richardson number, $N^{2}=\partial_{z} \bar{b}$ is the vertical

\footnotetext{
${ }^{1}$ Note that this also scales as 2 times the ratio of centripetal to Coriolis accelerations in the radial momentum equation (Shakespeare 2016).
}

stratification, and $\partial_{z} \mathbf{u}$ is the vertical shear of alongfront velocity $\mathbf{u}=(0, \bar{v}, 0)$. Note, however, that $\bar{v}$ now includes both geostrophic and ageostrophic components. Also note that in the limit $\mathrm{Cu} \rightarrow 0$ we recover the classical nondimensional criterion for symmetric instability given by Hoskins (1974) valid for flows in TWB [cf. Eq. (2)].

In summary, the criterion, $\Phi<0$ (or $\Phi^{\prime}<0$ ), states that the product of absolute angular momentum and Ertel PV must be positive for hydrodynamic stability but that instability will result if either (but not both) of these quantities is negative. Intuitively this makes sense since absolute angular momentum $L$ is a conserved quantity for axisymmetric flow and the Ertel PV $q$ is a conserved variable for inviscid, adiabatic flow. Thus, the criterion combines both conservation principles into a single expression. However, the sufficiency of the criterion could not have been known from such conservation principles without first considering arguments and efforts of scientists (Fjortoft 1950; Ooyama 1966; Cho et al. 1993) summarized in Part I.

\section{b. Symmetric instability with curvature: Implications and motivation}

A notable consequence of Eq. (8) is that an asymmetry can emerge between anticyclonic and cyclonic flow at low-gradient Richardson numbers (Part I). Recall: for cyclonic curved flow $\mathrm{Cu}>0$, while for anticyclonic curved flow $\mathrm{Cu}<0$. It follows that cyclonic fronts may be less stable than anticyclonic fronts for the same Ri and $|\mathrm{Ro}|$. This occurs in low stratification and large vertical shear, since it is here that the inverse gradient Richardson number is large and the baroclinic term in $\Phi^{\prime}$ is increasingly negative [i.e., the second term in Eq. (8)].

Our interpretation is that the absolute vorticity vector is tilted relative to its TWB state and that this tilting modifies the range of Ro and $\mathrm{Ri}$ permitted for stability. Since symmetric instability is expected to occur at low Richardson numbers (Stone 1966, 1970), it follows that symmetric instability is modified relative to our present-day understanding, as it is largely based on TWB (Thomas 2005; Taylor and Ferrari 2009, 2010; Thomas and Taylor 2010; Thomas et al. 2013). ${ }^{2}$ Thus, the main motivation for this second portion of our study is to examine outcomes of the criterion, focusing on the dynamical regime necessary for symmetric instability. This is largely driven by curiosity, but it may have profound implications for energy, buoyancy, and tracer budgets within the ocean. As an example, this could have implications for ocean biogeochemistry, including transport of tracers such as $\mathrm{Mn}, \mathrm{Fe}, \mathrm{CH}_{4}, \mathrm{H}_{2}$, and ${ }^{3} \mathrm{He}$ (Baker et al. 1995; Speer and Marshall 1995), and enhanced tracer exchange at the base of the ocean surface mixed layer, with corresponding implications for upper-ocean biology (Smith et al. 2016). These subjects are not addressed here, but they nonetheless motivate such a study.

\footnotetext{
${ }^{2}$ For other dynamical regimes in which gravitational or inertial/centrifugal instabilities principally occur, the reader is referred to classical convection and barotropic vortex studies (e.g., Imberger 1985; Kloosterziel and van Heijst 1991).
} 


\section{c. Outline of the study}

This second portion of our study is organized as follows. In section 2, we examine application of the nondimensional criterion to axisymmetric vortices in GWB, investigating interesting outcomes of the expression in nondimensional ( $\mathrm{Ro}, \mathrm{Ri}, \mathrm{Cu}$ ) parameter space. We first discuss idealized, base flows employed in the study and then present the results of applying the criterion [cf. Eq. (8)] to these models. In section 3, we compare these results with several observations, focusing on small-scale, coherent vortices in the ocean interior. By this, we refer to midlatitude submesoscale vortices and polar mesoscale vortices. Although dependent upon the base flow examined (through the local curvature-vorticity ratio, $\mu=\mathrm{Cu} / \mathrm{Ro}$ ), we nevertheless find our results provide a reasonable explanation for why coherent vortices are predominantly anticyclonic (McWilliams 1985, 2016). The argument of vortex tilting given in Part I essentially summarizes this interpretation. In section 4 , we discuss these results in light of recent studies and conclude the study in section 5. While our study is mostly idealized, it lays the groundwork for realistic, fine-scale modeling or observational studies investigating these topics. The reader is referred to a study by Shakespeare (2016) for additional discussions with regard to dynamics within curved density fronts.

\section{Criteria applied to curved baroclinic fronts and vortices}

Below, we consider implications of the nondimensional generalized Rayleigh criterion, $\Phi^{\prime}<0$, applied to curved fronts and vortices. In particular, we are interested in better understanding how $\mathrm{Cu}$ affects the range of Ro and $\mathrm{Ri}$ permitted for hydrodynamic stability. We do so using idealized inviscid, baroclinic vortices in GWB, ensuring that these base flows are characterized by low-gradient Richardson numbers.

We have found in the limit of low $\mathrm{Cu}$ (not shown) that the nondimensional Ertel PV for a flow in GWB $q^{\prime}$ does not smoothly approach the classical nondimensional discriminant of Hoskins (1974). We therefore do not encourage its use as a stability discriminant. Instead, we use $q^{\prime}$ [cf. Eq. (9)] only to better understand $\Phi^{\prime}=L^{\prime} q^{\prime}$. Note, for example, that it can inform us about what might be possible in an extreme case-that is, the non-axisymmetric case for which $L$ does not need to be conserved. In this manner, it proves informative for understanding how $L^{\prime}$ and $q^{\prime}$ separately affect the solution. A more appropriate investigation of the non-axisymmetric case should make use of the criterion developed by Billant and Gallaire (2005).

In summary, we consider three stability discriminants in the work that follows: 1) $q^{\prime}$ under TWB (as it helps to reveal when curvature becomes important), 2) $q^{\prime}$ under GWB (hereinafter referred to as a "non-axisymmetric discriminant"), and 3) $\Phi^{\prime}$ (the relevant stability discriminant).

\section{a. Methods}

To accomplish our main task, we simulate simple baroclinic flows in GWB using idealized vortex models. In all cases examined, the velocity structure of the vortex is assumed separable in the radial $r$ and vertical $z$ directions:

$$
\bar{v}=V(r) Z(z)
$$

where $\bar{v}$ is the azimuthal velocity at a location $(r, z)$ within the vortex, $V(r)$ denotes its horizontal structure, and $Z(z)$ defines the vertical structure of the vortex. Similarly, the vorticity has form $\bar{\zeta}=\omega(r) Z(z)$. In the discussion that follows, it is simplest to define the horizontal structure of the vortex, proceeding to the vertical structure second. We then describe how buoyancy and relevant gradients can be estimated from the velocity field. As we proceed, we encourage readers to bear in mind that these results are applicable to curved fronts in GWB so long as we confine our examination to the core of the vortex (i.e., $r<r_{m}$, where $r_{m}$ is the radius of maximum velocity).

\section{1) RANKINE VORTEX}

Although we do not use the classical Rankine vortex in our analysis, ${ }^{3}$ it helps to introduce the shielded Rankine vortex, below. The classical Rankine model consists of a core in solidbody rotation (i.e., $\mathrm{Ro}=\mathrm{Cu}$ ) within a radius $r_{m}$ and zero vorticity outside this distance (Kundu and Cohen 2008). At a fixed depth $|z|$, the velocity profile is given by

$$
V(r)=\left\{\begin{array}{ccc}
v_{m}\left(\frac{r}{r_{m}}\right), & \text { if } & r r_{m} \\
v_{m}\left(\frac{r}{r_{m}}\right)^{-1}, & \text { if } & r>r_{m}
\end{array},\right.
$$

with a corresponding vorticity

$$
\omega(r)=\left\{\begin{array}{cll}
\zeta_{o}, & \text { if } \quad r \leq r_{m} \\
0, & \text { if } \quad r>r_{m}
\end{array}\right.
$$

Here, $v_{m}$ is the maximum velocity (defined as positive for cyclonic flows), again, $r_{m}$ is the radius of maximum velocity, and we have defined a representative core vorticity as $\zeta_{o}=2 v_{m} / r_{m}$. (This corresponds to the maximum vorticity within all of the vortex models examined in our study.)

\section{2) SHIELDED RANKINE VORTEX}

Barotropic and baroclinic instabilities of ocean currents often form nonisolated vortices, meaning that their circulation is not independent of the background flow field (Lazar et al. 2013). One such example is the classical Rankine vortex presented above. However, in realistic numerical simulations, we often see vortices that are isolated from the background flow. Described as "shielded," these vortices consist of a ring or shield of oppositely signed vorticity surrounding a vortex core (Gallaire and Chomaz 2003; Kloosterziel et al. 2007). A simple model that crudely reflects this case is the shielded Rankine vortex. This base flow is useful in modeling intense vortices or those that may have recently been formed (Lilly and Rhines 2002; Timmermans et al. 2008).

The shielded Rankine vortex consists of a core in solid-body rotation (i.e., $\mathrm{Ro}=\mathrm{Cu}$ ) within a radius $r_{m}$, constant negative

\footnotetext{
${ }^{3}$ Integration from $r=0$ to $r=\infty$ will demonstrate that it does not converge, and we use such an integral in our calculations.
} 
vorticity outside this distance to a radius $b r_{m}(b>1)$, and zero vorticity elsewhere. Mathematically, this is easiest to express in terms of relative vorticity:

$$
\omega(r)=\left\{\begin{array}{cll}
\zeta_{o}, & \text { if } r \leq r_{m} \\
-\zeta_{o}\left(b^{2}-1\right)^{-1}, & \text { if } \quad r_{m}<r \leq b r_{m} \\
0, & \text { if } r>b r_{m}
\end{array}\right.
$$

where the velocity at a fixed depth $|z|$ is related to the vorticity $\omega(r)$ at that same depth as

$$
V(r)=\frac{1}{r} \int_{0}^{\infty} r \omega(r) d r .
$$

All isolated or shielded vortices are susceptible to barotropic shear instability (Flierl 1988), with narrower shields being more unstable. To minimize sensitivity to horizontal shear, we set $b=4$, yielding a shield with thickness $3 r_{m}$. In the limit of $b \rightarrow \infty$, the shielded Rankine vortex is equivalent to the classical Rankine vortex.

\section{3) AlPHA-EXPONENTIAL VORTEX}

We additionally examine the stability of a family of shielded vortices introduced by Carton and McWilliams (1989) but investigated prior to this by X. Carton and B. Legras (1988, unpublished manuscript). This is sometimes referred to as the generalized Gaussian vortex, since the Gaussian vortex is obtained as a special case, or simply the $\alpha$-exponential vortex. Hereinafter, we refer to this as the Carton-Legras-McWilliams (CLM) vortex. Work by Gallaire and Chomaz (2003), among many others, serves as a useful reference for this vortex class.

The CLM vortex has a velocity structure defined by ${ }^{4}$

$$
V(r)=v_{m}\left(\frac{r}{r_{m}}\right) \exp \left[-(1 / 2)\left(r / r_{m}\right)^{\alpha}\right]
$$

with corresponding vorticity given by

$$
\omega(r)=\zeta_{o}\left[1-\frac{\alpha}{4}\left(\frac{r}{r_{m}}\right)^{\alpha}\right] \exp \left[-(1 / 2)\left(r / r_{m}\right)^{\alpha}\right] .
$$

In these expressions, $\alpha$ is a "steepness parameter" that controls how quickly the velocity decays away from its value at $r=r_{m}$. One notes that the classical Gaussian vortex (i.e., Gaussian in vorticity) is obtained by setting $\alpha=2$ and that typical values for the oceanic environment are $1.5 \leq \alpha \leq 2.5$.

The Gaussian model $(\alpha=2)$ strikes a unique balance between possessing a negative vorticity shield while being only weakly unstable to barotropic shear (Flierl 1988). It has proven useful in depicting less intense vortices, including those at larger scales (Chelton et al. 2011) or that may have translated far from their origin (Paillet et al. 2002). A negative aspect of

\footnotetext{
${ }^{4}$ Most definitions neglect the factor of $1 / 2$ in the velocity (Gallaire and Chomaz 2003), because it leads to a cleaner expression for vorticity $\omega(r)$. Although both are valid, we retain the scale factor to ensure that, in the Gaussian vortex $(\alpha=2)$, the vorticity is nonzero at $r=r_{m}$.
}

this model, however, is that it cannot accurately depict intense vortices, failing to arrive at large vorticity values typically seen in submesoscale observations, for instance. For this reason, the Rankine vortex (or some variant thereof) or the CLM vortex with $\alpha \geq 2.5$ is appropriate when modeling intense vortices.

In our study, we report results for the CLM vortex with $\alpha=$ $1.5,2.0$, and 2.5 , focusing on the vortex with $\alpha=2.5$ when comparing with observations (section 3 ). We do not provide observational support for one vortex type over another and, because of this, our study is inconclusive. Nevertheless, as there is growing support for a universal form to vortices in rotating, stratified flows (Aubert et al. 2012; Zhang et al. 2013; Mahdinia et al. 2017), this selection of base flows appears reasonable.

\section{4) VERTICAL STRUCTURE}

In all cases, the vertical structure is modeled as Gaussian, which is a reasonable approximation for oceanic vortices (e.g., Riser et al. 1986; McWilliams 1985; Paillet et al. 2002; Timmermans et al. 2008):

$$
Z(z)=\exp \left[-\left(z-z_{o}\right)^{2} / h^{2}\right]
$$

where $h=H / 2$ is the $e$-folding scale of the eddy, or half the vertical scale. We model the flow as having an inviscid boundary at the surface $\left(z_{o}=0\right)$, although it is straightforward to extend this to a subsurface vortex by reflecting the results presented here about the topmost boundary. Buoyancy gradients differ in top and bottom portions of the eddy, but nondimensional numbers remain the same. McWilliams (1985), for example, presents only the top half of a vortex. Typical values of $H$ for oceanic submesoscale vortices are 100-200 m.

\section{5) ENSURING PROPER BALANCE}

The vortex in GWB has a unique density structure that must be satisfied for proper balance. There are probably several ways to ensure balance, but the simplest is to start with the velocity field defined above, $\bar{v}=V(r) Z(r)$ [cf. Eq. (10)]. Vertical differentiation of $\bar{v}$ yields

$$
\partial_{z} \bar{v}=V \partial_{z} Z=\left(-\frac{2 z}{h^{2}}\right) V Z .
$$

We can then use GWB [cf. Eq. (4)] to relate the vertical shear to the lateral buoyancy gradient:

$$
\partial_{r} \bar{b}_{a}=\left(f+\frac{2 \bar{v}}{r}\right) \partial_{z} \bar{v}=\left(-\frac{2 z}{h^{2}}\right)\left(f+\frac{2 V Z}{r}\right) V Z .
$$

Next, integrating radially inward, ${ }^{5}$ we obtain the buoyancy anomaly,

$$
\bar{b}_{a}=\int_{\infty}^{0}\left(\partial_{r} \bar{b}_{a}\right) d r=\left(\frac{2 z}{h^{2}}\right) \int_{0}^{\infty}\left(f+\frac{2 V Z}{r}\right) V Z d r,
$$

\footnotetext{
${ }^{5}$ The reason for integrating radially inward is that we can specify the lateral buoyancy gradient at the most distant part of the domain to be zero.
} 
demonstrating that the buoyancy anomaly is directly related to the integral of the velocity structure. Vertical differentiation of Eq. (20) gives the associated anomalous stratification, $N_{a}^{2}=\partial_{z} \bar{b}_{a}$.

We then embed the vortex into a constant background buoyancy gradient $N_{b}^{2}=\partial_{z} \bar{b}_{b}$ such that the total stratification at any point $(r, z)$ is given by $N^{2}=\partial_{z} \bar{b}=N_{b}^{2}+N_{a}^{2}=\partial_{z} \bar{b}_{b}+\partial_{z} \bar{b}_{a}$. From velocity and buoyancy gradients, we estimate nondimensional numbers ( $\mathrm{Ro}, \mathrm{Ri}$, and $\mathrm{Cu}$ ) and assess the stability of the flow. In all cases, we use centered differences to estimate the derivatives.

\section{6) Practical CONSIDERATions}

Our domain is specified as $r / r_{m}=[0,10]$ and $z / H=[0,1]$, with $z / H=0$ corresponding to the topmost boundary (midheight of an eddy for a subsurface eddy). This radial domain size is sufficient to ensure convergence of the above integral. The variables $v_{m}, r_{m}$, and nondimensional stratification $N / f$ were then varied so as to cover the desired parameter space. We varied the nondimensional background stratification $N_{b} / f$ over the range 10-100 in an effort to populate the parameter space at low Richardson numbers. Also, we at first varied $H$ but quickly found that a large range of $\mathrm{Ri}$ was achieved by varying the other parameters. We implemented these simple simulations using 400 points in the vertical direction and 500 points in the horizontal plane, fixing the Coriolis parameter as $f=10^{-4} \mathrm{~s}^{-1}$ for all simulations.

Rossby and curvature numbers, Ro and $\mathrm{Cu}$, are not independent. At a given location within a front, they are linearly related. As a result, the stability curve $\Phi^{\prime}$ resides on a plane in ( $\mathrm{Ro}, \mathrm{Ri}, \mathrm{Cu}$ ) space. In displaying our results, we have chosen to first depict the three-dimensional stability map and then illustrate $\Phi^{\prime}$ as a function of Ro ( $y$ axis) and Ri ( $x$ axis). We also illustrate as a separate axis (in red font) curvature numbers corresponding to each gradient Rossby number.

In summary, we examine three stability discriminants in the work that follows: (i) the nondimensional Ertel PV $q^{\prime}$ under TWB [cf. Eq. (2)] since it helps to demonstrate when curvature effects become important, (ii) the nondimensional Ertel PV $q^{\prime}$ under GWB [cf. Eq. (9)] since it serves as an input to $\Phi^{\prime}$, and (iii) the nondimensional Rayleigh discriminant $\Phi^{\prime}\left(=L^{\prime} q^{\prime}\right)$ [cf. Eq. (8)]. Note, that the nondimensional absolute angular momentum $L^{\prime}=1+\mathrm{Cu}$, can be readily visualized in terms of $\mathrm{Cu}$ so that it is not necessary to display this quantity.

\section{b. Results}

We now present the results, first illustrating relevant dynamics with simple anticyclonic and cyclonic Rankine vortices (Figs. 1-8) and, second, summarizing results over the full nondimensional parameter space (i.e., stability maps) for all vortices (Figs. 9-12).

\section{1) Anticyclone}

We first consider the case of an anticyclonic vortex. Figures 1-3 illustrate the result of the analysis applied to an anticyclonic vortex. Here, the vortex is specified to have a maximum velocity of $v_{m}=-0.5 \mathrm{~m} \mathrm{~s}^{-1}$ and radius of maximum velocity $r_{m}=10 \mathrm{~km}$, yielding $\mathrm{Ro}=\mathrm{Cu}=-1$ at $r / r_{m}=1$. The gradient Richardson number, Ri, was chosen to be low (i.e., $\mathrm{Ri} \sim 1.5$ in the lowest region) in order to illustrate the interplay between $\mathrm{Ro}$ and $\mathrm{Cu}$ in terms of its effect on PV. Figure 4 summarizes the difference obtained when accounting for curvature. Under the classical instability criterion (Hoskins 1974), we find that the flow is unstable in the vortex core (cf. Fig. 4a). However, when accounting for the curvature of the front, we observe that the flow is stable (cf. Fig. 4b). Moreover, even in the axisymmetric discriminant (cf. Fig. 4c), the stabilization of the vortex persists, though this increase in stability is minimal. Nevertheless, it reveals that stabilization of anticyclones is possible for both axisymmetric and non-axisymmetric vortices. This illustrates an important finding: anticyclonic flows with large Rossby numbers can be stable if the curvature is large and gradient Richardson number is low.

\section{2) CyClone}

We next consider the case of a cyclonic vortex. Figures 5-7 illustrate these diagnostics applied to a cyclonic vortex. In this case, the vortex is specified to have maximum flow speed $v_{m}=$ $0.5 \mathrm{~m} \mathrm{~s}^{-1}$ and radius of maximum velocity $r_{m}=3 \mathrm{~km}$, giving $\mathrm{Ro}=\mathrm{Cu}=3.3$ at $r / r_{m}=1$. The gradient Richardson number, $\mathrm{Ri}$, is again low (e.g., $\mathrm{Ri} \approx 2$ in the lowest region) in order to illustrate the compensation of the vorticity and curvature. The vortex core is stable, as expected for equal $\mathrm{Ro}=\mathrm{Cu}$ but with $\mathrm{Ri}>1.5$. Examining the region just beyond the core of the vortex (Fig. 7), we find that as the distance exceeds $r / r_{m}=1$, the curvature number remains large while the vorticity falls precipitously. This is the reason for the low PV near $r / r_{m}=1$. With increased $r / r_{m}$, the vertical shear reduces and the curvature number relaxes, making PV positive once again. Realistic vortices such as the Gaussian vortex will encounter this change in a less abrupt manner. Nevertheless, this particular vortex illustrates in a clear manner the potential impact of such differences in $\mathrm{Ro}$ and $\mathrm{Cu}$ within a vortex.

The importance of the curvature can be seen more readily by comparing all three discriminants (Fig. 8). Here, we display $q^{\prime}$ valid for TWB (Fig. 8a), $q^{\prime}$ valid for GWB (Fig. 8b), and $\Phi^{\prime}$ valid for axisymmetric flow in GWB (Fig. 8c). As in the anticyclonic case, a difference between these discriminants exists for the cyclonic vortex. In particular, the flow is stable under the classical criterion (Fig. 8a), but it is unstable both for nonaxisymmetric and axisymmetric cases (Figs. 8b,c). In contrast to the anticyclonic case where curvature stabilized the flow, here it reduces stability. This illustrates a second important finding: cyclonic flows with curvature are more prone to instability than might be expected from the instability criterion of Hoskins (1974). This is a robust feature of both nonaxisymmetric and axisymmetric vortices.

\section{3) FULL NONDIMENSIONAL PARAMETER SPACE}

To better understand conditions under which curvature becomes important, we repeated the calculations presented above for a wide range of azimuthal velocities, radii, and stratification. In this study, we display results for the shielded Rankine and CLM vortices ( $\alpha=1.5,2.0$, and 2.5). It is sufficient for our present purpose to study a single location within 

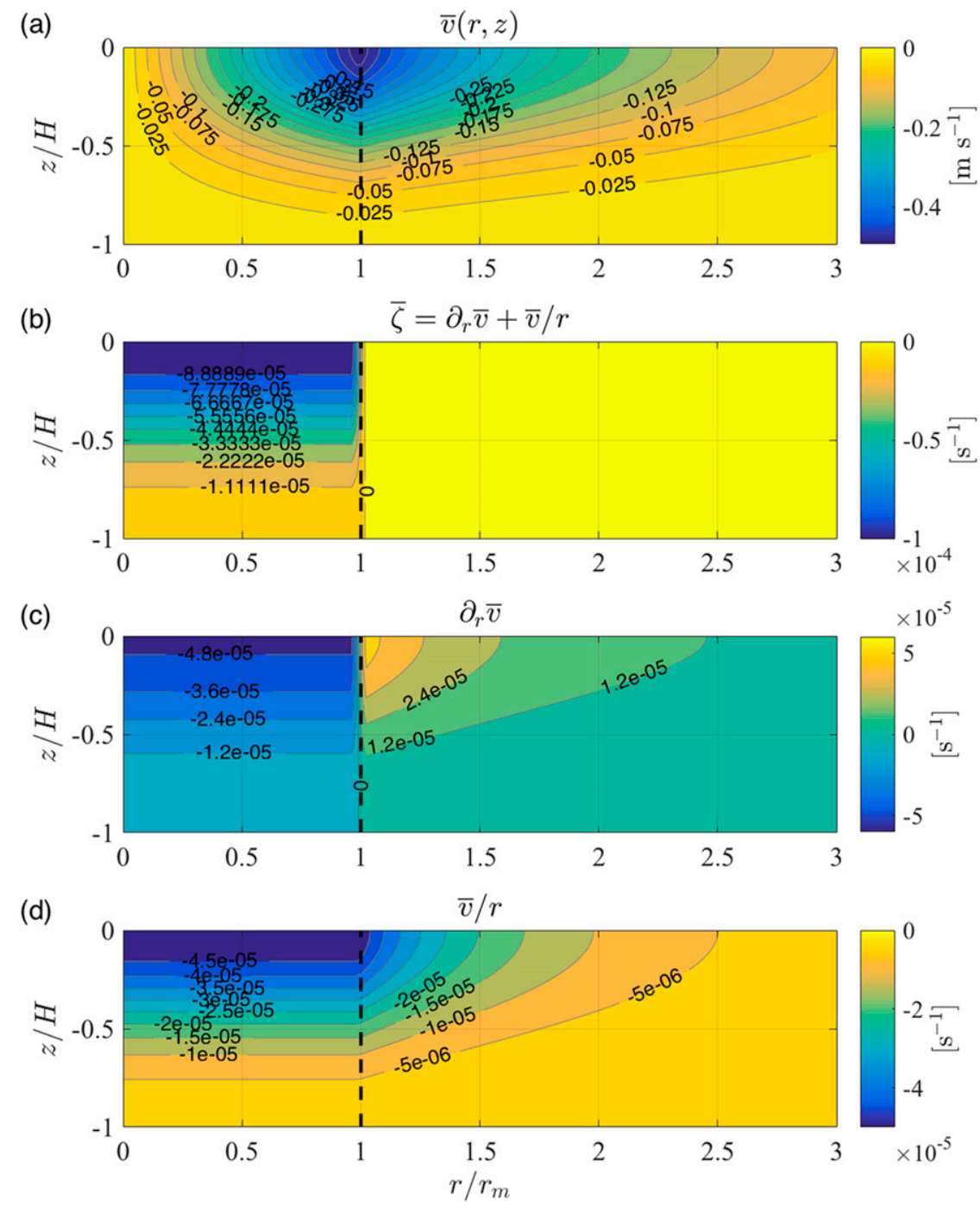

FIG. 1. Velocity structure within an anticyclonic vortex: (a) velocity and (b) relative vorticity and (c),(d) corresponding components of relative vorticity. This particular example corresponds to a shielded Rankine vortex with maximum velocity $v_{m}=-0.50 \mathrm{~m} \mathrm{~s}^{-1}$, radius of maximum velocity $r_{m}=10 \mathrm{~km}$, and shield parameter $b=4$. Here and in other figures, a contour line with an "e" label indicates that the numeral preceding the e should be multiplied by 10 raised to the sign and numerals following the e.

the vortex. The choice of location would ideally be characterized by vorticity of the same sign as the bulk Rossby number $\mathrm{Ro}_{b}=v_{m} /\left(f r_{m}\right)$. However, this choice is complicated by the fact that unstable regions of cyclones and anticyclones slightly differ: cyclones are unstable just outside $r_{m}$ whereas anticyclones are approximately unstable in the vortex core (see examples above). However, a compromise can be made by noting that symmetric and inertial instabilities often have some breadth to them (e.g., Kloosterziel et al. 2007, their Fig. 3). Thus, for this study we have chosen to examine the location, $\left(r / r_{m}, z / H\right)=(1,-1 / 2)$, allowing us to observe the stability of both cyclonic and anticyclonic flows at the same time. In the next subsection, we examine a "global average" or an average over the vortex to see whether the results found at our local study location translate to bulk Rossby numbers characterizing the whole of the front. A more thorough understanding requires a global stability analysis such as the ones performed by Lahaye and Zeitlin (2015) and Mahdinia et al. (2017), or a fully nonlinear analysis.

The stability results for the shielded Rankine vortex are displayed in Fig. 9, while those for the CLM vortex are shown in Figs. 10-12. In these figures, we display $q^{\prime}$ under TWB (panels a and b), $q^{\prime}$ under GWB (panels $\mathrm{c}$ and d), and the generalized Rayleigh discriminant $\Phi^{\prime}$ (panels e and f), where negative values indicate instability. At low Ro and low Ri, we have a reduced number of simulated anticyclones. This occurs because, even in these weak background stratifications $(N / f \sim$ 10 to 100$)$, anticyclones flow are associated with elevated $\mathrm{Ri}$ 


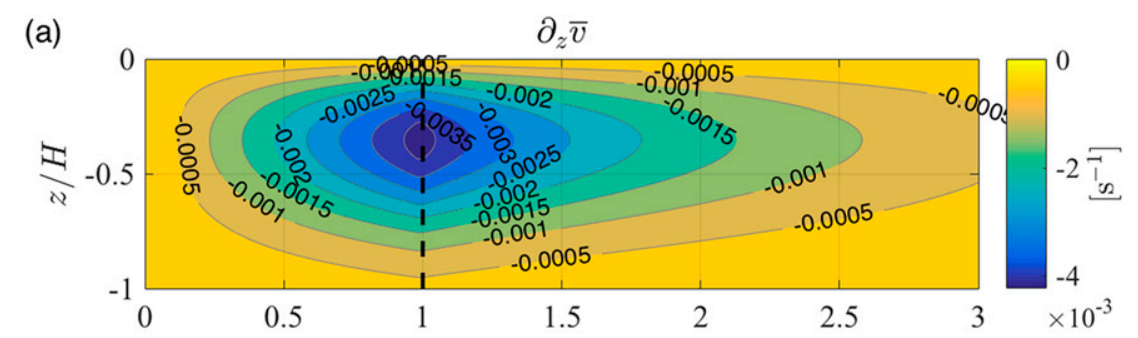

(b)
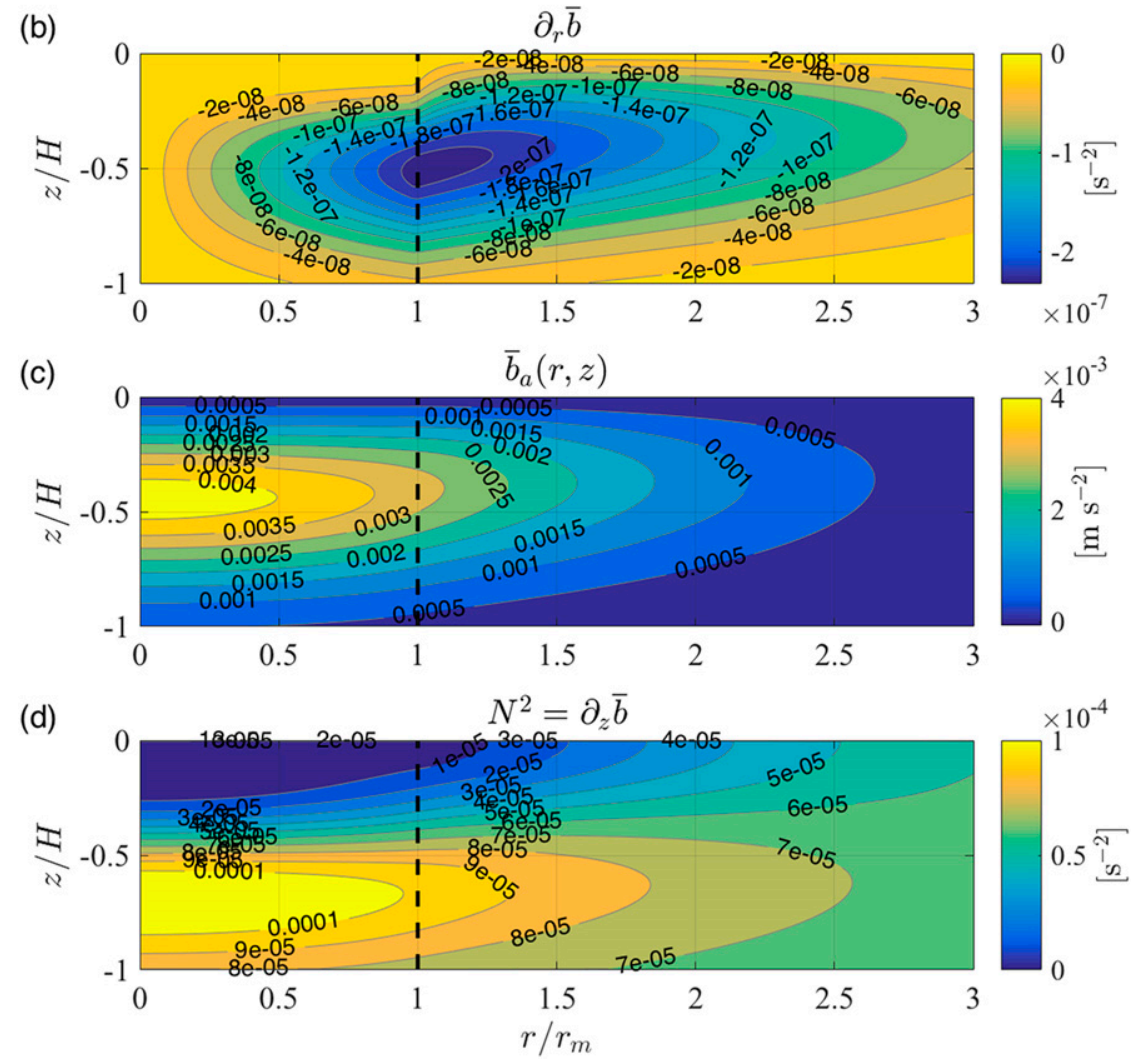

FIG. 2. Baroclinic structure of an anticyclonic vortex: (a) vertical shear, (b) lateral buoyancy gradient, (c) associated buoyancy anomaly, and (d) vertical stratification. Here, vertical stratification is computed as the sum of a background stratification $\left(N / f=80\right.$, with $\left.f=10^{-4} \mathrm{~s}^{-1}\right)$ and anomalous stratification associated with the vortex.

at $r / r_{m}=1$ when compared with cyclones. This is particularly evident for CLM vortices with $\alpha \leq 2.0$ and the Rankine vortex.

To begin, we first examine the Ertel PV for flows in TWB (top row) and GWB (middle row). The first quantity serves as the stability discriminant expected for straight fronts. We observe an increase in nondimensional PV (i.e., $q^{\prime}$ ) for anticyclonic flows in GWB for gradient Richardson numbers near unity (middle row). Again, this is counter to the more classical instability criterion, which predicts instability for anticyclonic flows with $|\mathrm{Ro}|$ close to unity (top row). Also evident is a reduction in PV for cyclonic flow characterized by gradient Richardson numbers near unity. This reduction in PV is not predicted by the Hoskins (1974) expression (top row) and, for CLM vortices, occupies a greater portion of the parameter space as the steepness parameter, $\alpha$, increases in magnitude $(\alpha \geq 2.0)$.
We now examine stability predicted by the Rayleigh discriminant $\Phi^{\prime}$ (bottom row). We observe three distinct features. First, owing to the multiplication of the nondimensional PV by $L^{\prime}=1+\mathrm{Cu}$, the front can become unstable for values $L^{\prime}<0$. As noted above, this approximately corresponds to bulk Rossby numbers of $\mathrm{Ro}_{b} \sim 0.5$ and gradient Rossby numbers of Ro $<-0.45$ to -0.7 , but the latter number depends on the vortex model examined. Second, because $1+\mathrm{Cu}>1$ for cyclonic flow, one finds that $\Phi^{\prime}>q^{\prime}$ and the destabilization of cyclones is always a robust characteristic of both axisymmetric (bottom panel) and nonaxisymmetric (middle panel) vortices. Third, the stability of anticyclones is reduced for the axisymmetric case when compared with the non-axisymmetric case but nevertheless remains positive. Of course, this may be specific to our study 

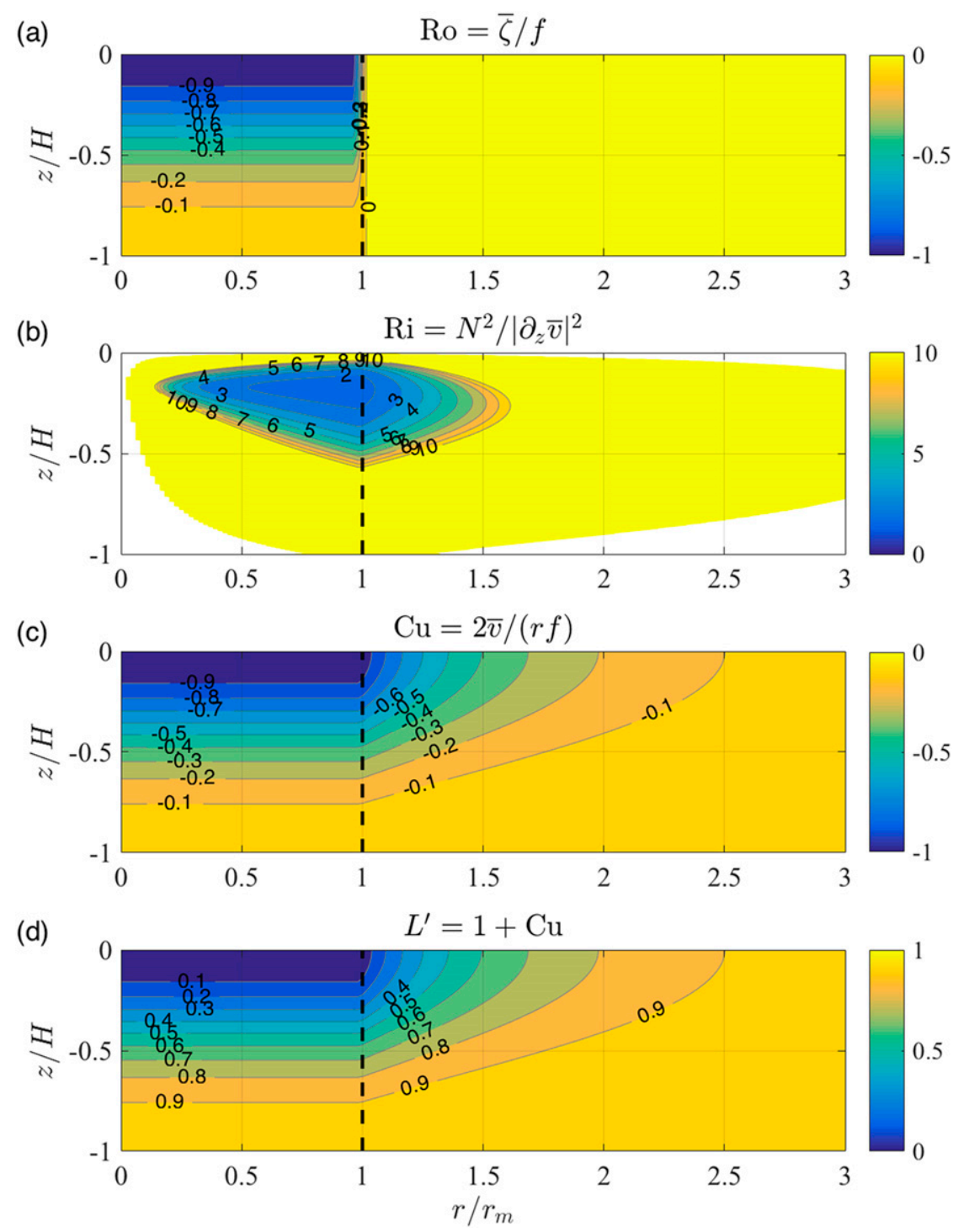

FIG. 3. Nondimensional parameters associated with an anticyclonic vortex: (a) gradient Rossby number, (b) gradient Richardson number, (c) curvature number, and (d) nondimensional absolute angular momentum $L^{\prime}=2 L /\left(f r^{2}\right)=1+\mathrm{Cu}$, where $L=r \bar{v}+f r^{2} / 2$ is the absolute angular momentum.

location. To determine if this is so, we also consider other locations within the vortex core.

\section{4) DEPENDENCE OF RESULTS ON LOCATION}

As is evident in the preceding stability maps, a linear relationship exists between curvature and vorticity. For a given vortex model and for a given location, $(r, z)$, within the curved axisymmetric front, we see that the ratio of $\mathrm{Cu}$ to $\mathrm{Ro}$ is constant. Moreover, this ratio, $\mu=\mathrm{Cu} / \mathrm{Ro}$, is constant with depth and is the same for cyclones and anticyclones. This information can be used to assess the stability of the front or vortex in other locations within the vortex core.

Focusing on the CLM vortex model with $\alpha=2.5$, we examined $\mu$ at numerous points within the domain (Fig. 13a).
Next, we generated evenly spaced grids of Rossby and Richardson numbers, related $\mathrm{Cu}$ to Ro through the curvaturevorticity relationship, and determined stability via Eq. (8). As the ratio, $\mu$ can be large, both positively and negatively, within the vortex shield and since we do not know to what degree observed vortices are characterized by such shields, we avoid analysis in this location. In essence, we are focusing on recently generated vortices whose core velocity and vorticity structure resembles that of the CLM vortex with $\alpha=2.5$, or curved fronts with these same curvature and vorticity properties.

Figure $13 \mathrm{~b}$ depicts the stability map evaluated locally at our study location (white circle in Fig. 13a). This graphic can be compared with Fig. 12f, which was generated with simulated vortices in GWB. There is no significant difference in these two 

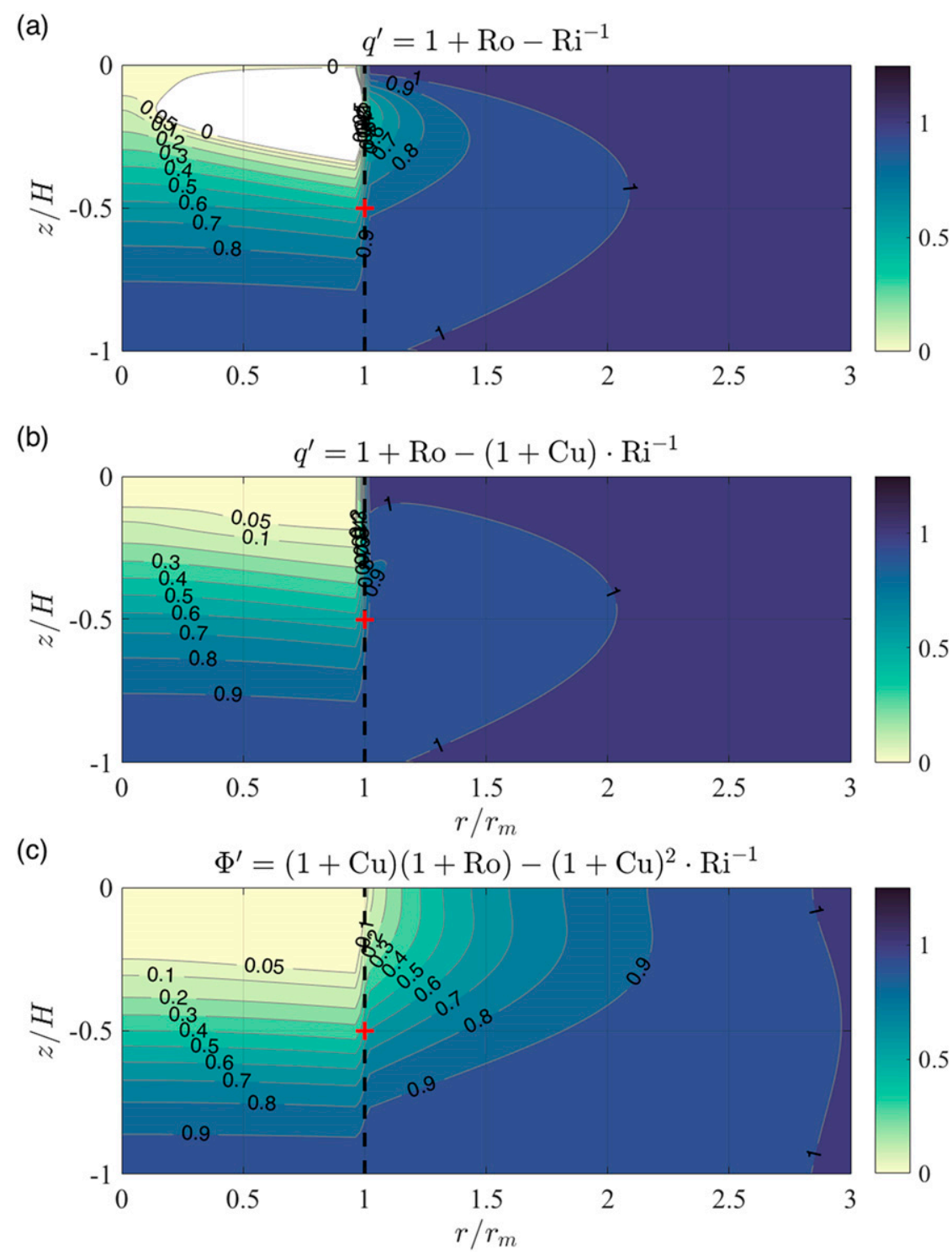

FIG. 4. Stability discriminants for an anticyclonic vortex for which negative values (white) indicate instability: (a) classical discriminant of Hoskins (1974) valid for baroclinic flow in thermal wind balance [TWB; cf. Eq. (2)], (b) nondimensional Ertel PV valid for baroclinic flow in GWB (cf. Eq. (9)], and (c) generalized Rayleigh discriminant valid for baroclinic flow in GWB [cf. Eq. (8)]. Red plus signs highlight the location used to fill the parameter space in Figs. 9-12, below.

stability maps except that the sample set for anticyclonic flow is now filled at low Ri. In Fig. 13c, we display the likelihood of observing stable states as a function of $|\mathrm{Ro}|$ for low Richardson numbers $(\mathrm{Ri}<2)$. Note that the probability of observing a stable anticyclone is considerably larger than the probability of observing a stable cyclone for $|\mathrm{Ro}|<0.5$-almost a factor of 2 in some instances.

To obtain an estimate of $\Phi^{\prime}$ representative of the vortex as a whole, we computed the global average, $\left\langle\Phi^{\prime}\right\rangle$, as shown in Fig. 13d, where we have weighted each location equally. The contours of the marginally stable state are taken from Fig. 13b to aid in comparison. First, the stability map for the averaged $\Phi^{\prime}$ (Fig. 13d) is comparable to the locally valid $\Phi^{\prime}$ (cf. Fig. 13b). Slight differences are evident at 1) low Richardson number $(\mathrm{Ri}<2)$ where anticyclonic flow has reduced stability and 2$)$ at elevated Richardson numbers near the marginally stable state, $\mathrm{Cu}=-1$. Overall, however, this global average approximately reflects the local stability map. This suggests locally valid stability maps presented earlier are indicative of the mean stability of the vortex. The histogram of stable states (Fig. 13e) depicts a slightly different relationship. Though a larger number of stable anticyclonic occurrences when compared with 

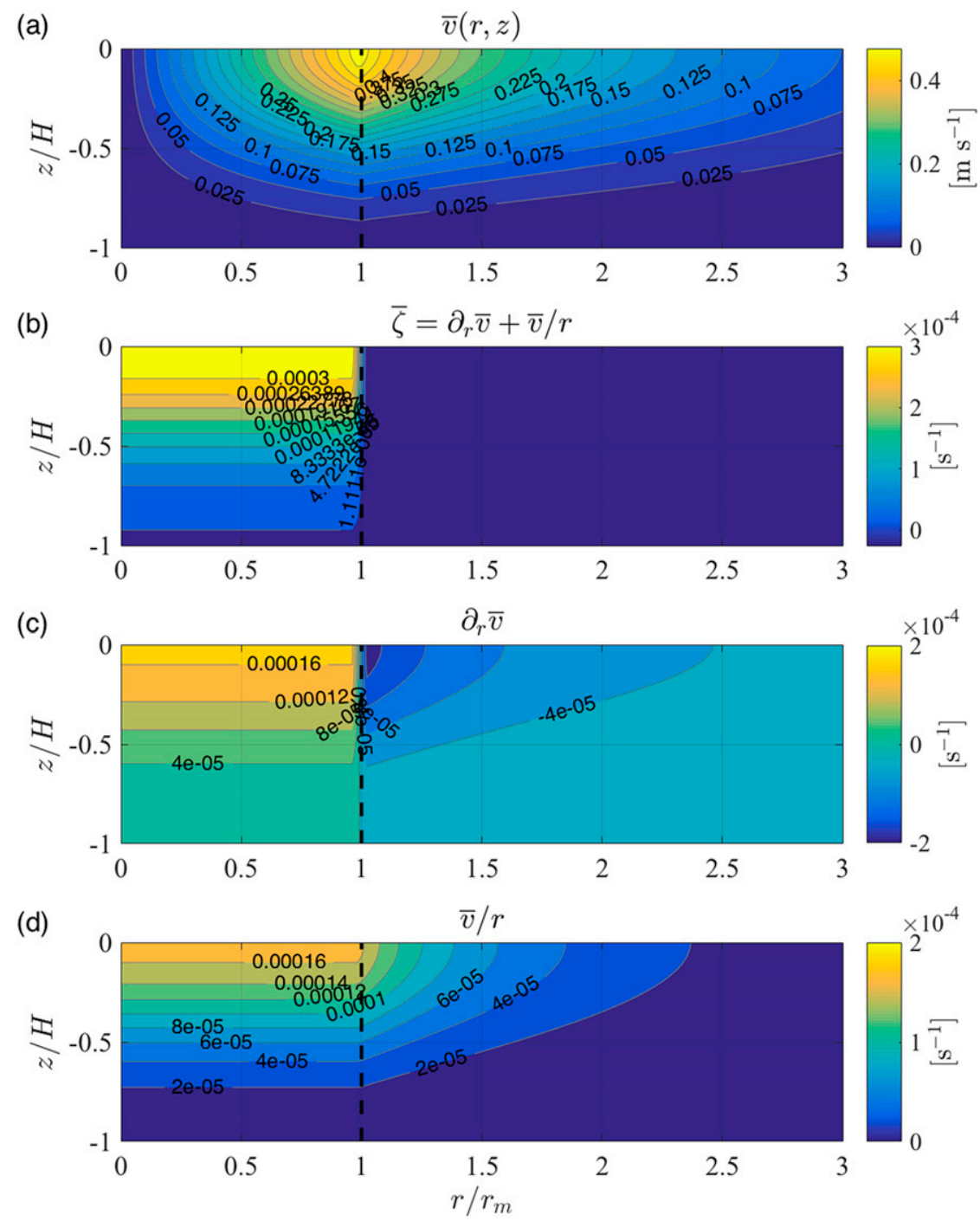

FIG. 5. As in Fig. 1, but for a cyclonic vortex. This particular example corresponds to a shielded Rankine vortex with maximum velocity $v_{m}=0.50 \mathrm{~m} \mathrm{~s}^{-1}$, radius of maximum velocity $r_{m}=3 \mathrm{~km}$, and shield parameter $b=4$.

stable cyclonic occurrences remain, the difference between these two groups is reduced. This suggests that anticyclones become less stable closer to their cores, $r<r_{m}$, a finding more consistent with inertial rather than symmetric instability.

Although we might have seen the possibility of synthetically generating the stability maps from the outset, there is merit in using the idealized baroclinic vortices since they provide a useful tool for better understanding the dynamics of vortices (Figs. 1-8). To this end, we include open-source Julia code (https://julialang.org/) as online supplemental material to help the reader to investigate some of these ideas.

\section{5) Marginal Stability CURVES}

Useful statements about stability of curved baroclinic fronts can obtained by substituting $\mu=\mathrm{Cu} / \mathrm{Ro}$ into the expression $\Phi^{\prime}=0$ [cf. Eq. (8)] and solving for Ro. One finds, for example, that Ro values corresponding to stable states $\left(\Phi^{\prime}>0\right)$ reside between two curves, which are the roots of the quadratic expression in Ro. The roots are $\mathrm{Ro}_{0}=-\mu^{-1}$ (corresponding to the curve $\mathrm{Cu}=-1)$ and $\mathrm{Ro}_{1}=(1-\mathrm{Ri}) /(\mathrm{Ri}-\mu)$ (corresponding to the hyperbolic curve that asymptotes at $\mathrm{Ri}=\mu$ ). We refer to $\mathrm{Ro}_{0}$ and $\mathrm{Ro}_{1}$ as barotropic and baroclinic roots, respectively, since they highlight different aspects of the stability discriminant [Eq. (17) in Part I]. Both marginal stability curves are highlighted in Fig. $13 \mathrm{~b}$, for which $\mu \approx 2$. Note that, while both constrain gradient Rossby numbers permitted for stable flow, the baroclinic root helps explain the dominance of anticyclonic flow found at low Richardson numbers, $\mathrm{Ri}<\mu$. This root is discussed below in section 4. It is also useful to note that $\mu$ can be recast in terms of strain rate: $\mu=1-\left(S_{s} / f\right) / \mathrm{Ro}$, where $S_{s} / f=\partial_{r} \bar{v} / f-\bar{v} /(f r)=\mathrm{Ro}-\mathrm{Cu}$ is a nondimensional shear component of strain. We therefore expect greater 

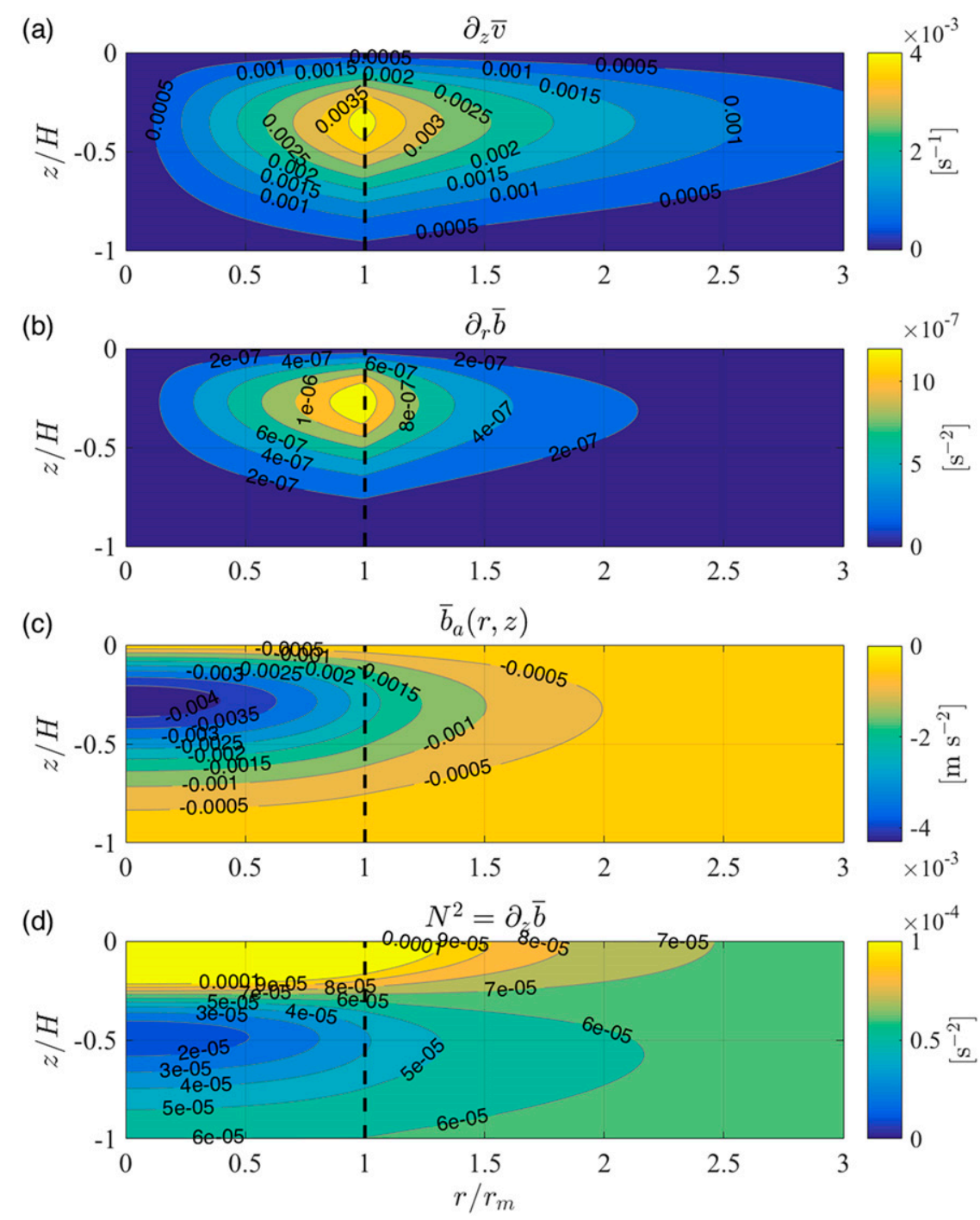

FIG. 6. As in Fig. 2, but for a cyclonic vortex. As before, $N / f=80$, with $f=10^{-4} \mathrm{~s}^{-1}$.

skewness when $\mu>1$, or when $S_{s} / f$ has sign opposite that of Ro. This typically happens near $r=r_{m}$, which is consistent with elevated curvature-vorticity ratios found at these radii (cf. Fig. 13a). Thus, an alternative way of examining these marginal stability curves is in terms of strain rate (T. Meunier 2020, personal communication). This, we leave for a future study.

\section{Comparison with observations}

It is worth considering how these results extend to curved fronts and vortices in the observed ocean. Application of our results to mesoscale fronts and vortices at mid- and subpolar latitudes (Chelton et al. 2011; Frenger et al. 2015) may be limited owing to the smallness of curvature numbers for such flows. This was found to be the case, for example, in a study of instabilities on the edge of a Southern Ocean eddy (Adams et al. 2017). It is notable, however, that the generalized Rayleigh criterion $\Phi<0$ was not used in that study. This said, Chelton et al. (2011) documented an observed asymmetry in the distribution of long-lived vortices (lifetimes $>40$ weeks) observed from satellite altimetry (Chelton et al. 2011, Fig. 2). Thus, it is possible that symmetric instability found within mesoscale eddies preferentially erodes cyclones when compared with anticyclones as they are exposed to strong wintertime cooling. This would be an interesting avenue to explore.

An obvious exception to the statement about the smallness of the curvature number can be found at low latitudes. As noted by Shakespeare (2016), the Coriolis parameter is reduced but nonzero and horizontal velocities can be considerable, e.g., $0.3 \mathrm{~m} \mathrm{~s}^{-1}$ (Holmes et al. 2014), thereby making $\mathrm{Cu}$ nonnegligible. However, the increase in the meridional component of Coriolis as one approaches the equator ${ }^{6}$ might

\footnotetext{
${ }^{6}$ The relevant nondimensional parameter is $N / \tilde{f}$, where $\tilde{f}=2 \Omega \cos \theta$ is the meridional component of the full Coriolis vector, $2 \Omega=(0, \tilde{f}, f)$. For values $N / \tilde{f}<5$, one should consider such dynamics (Colin de Verdière 2012).
} 

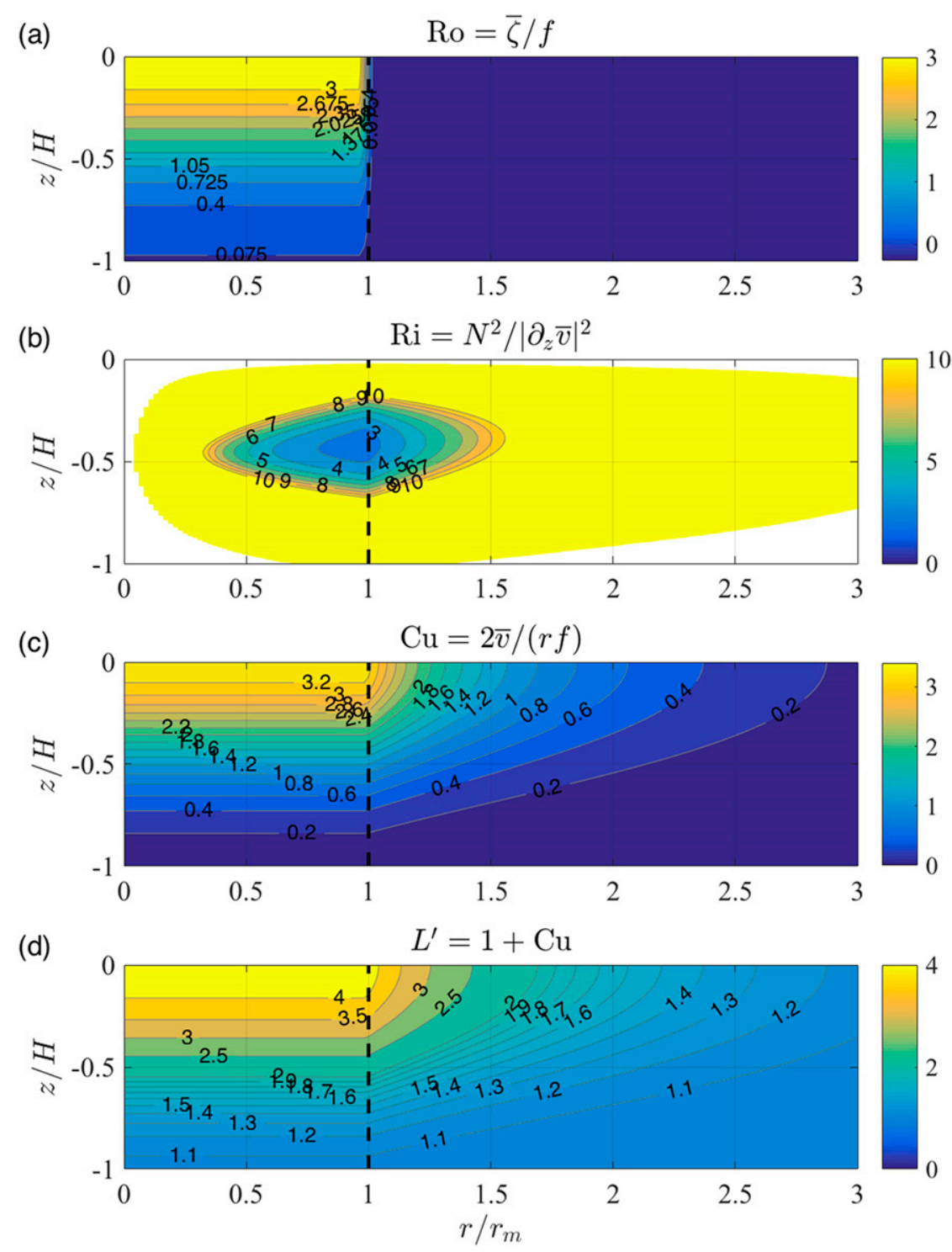

FIG. 7. As in Fig. 3, but for a cyclonic vortex.

complicate interpretation by invalidating our starting assumptions (Appendix A in Part I). This point has also been raised by Colin de Verdière (2012), Kloosterziel et al. (2017), and Zeitlin (2018) in the context of symmetric instability. For these reasons, perhaps the clearest extension of our results is in better understanding small-scale, coherent vortices, including midlatitude submesoscale and polar mesoscale vortices, or eddies.

\section{a. Small-scale, coherent vortices in the ocean}

McWilliams (1985) provided a comprehensive description of coherent vortices in the ocean interior. By this, we mean vortices that may have formed within boundary layers and yet are observed somewhat distant from these boundaries. Termed submesoscale coherent vortices (SCVs), these phenomena were differentiated from other oceanic eddies by their dynamics, e.g., Ro $\sim O(1)$, and also by their vertical positions within the oceans-e.g., thermocline, subthermocline, and meddy, where the name of the latter class of eddies refers to its expected origin deduced from water mass properties (McDowell and Rossby 1978). With lifetimes on the order of months to years, the anomalies are evident as deviations in density, velocity, salinity, or chemical composition (e.g., oxygen). The breadth or diameter of the vortices ranges between 5 and $50 \mathrm{~km}$, with typical core radii on the order of $10 \mathrm{~km}$. A common vertical scale is $H=200 \mathrm{~m}$. Additionally, an overwhelming majority of these vortices are anticyclonic (Bane et al. 1989) and a distinguishing feature of the SCV's vertical structure is its low vertical stratification. For example, values of $10<N / f<65$ are common (Table 1 ). Moreover, they have well-mixed water properties within their interior suggesting homogenization through turbulent mixing processes. While sample size is limited, the following observations may provide 

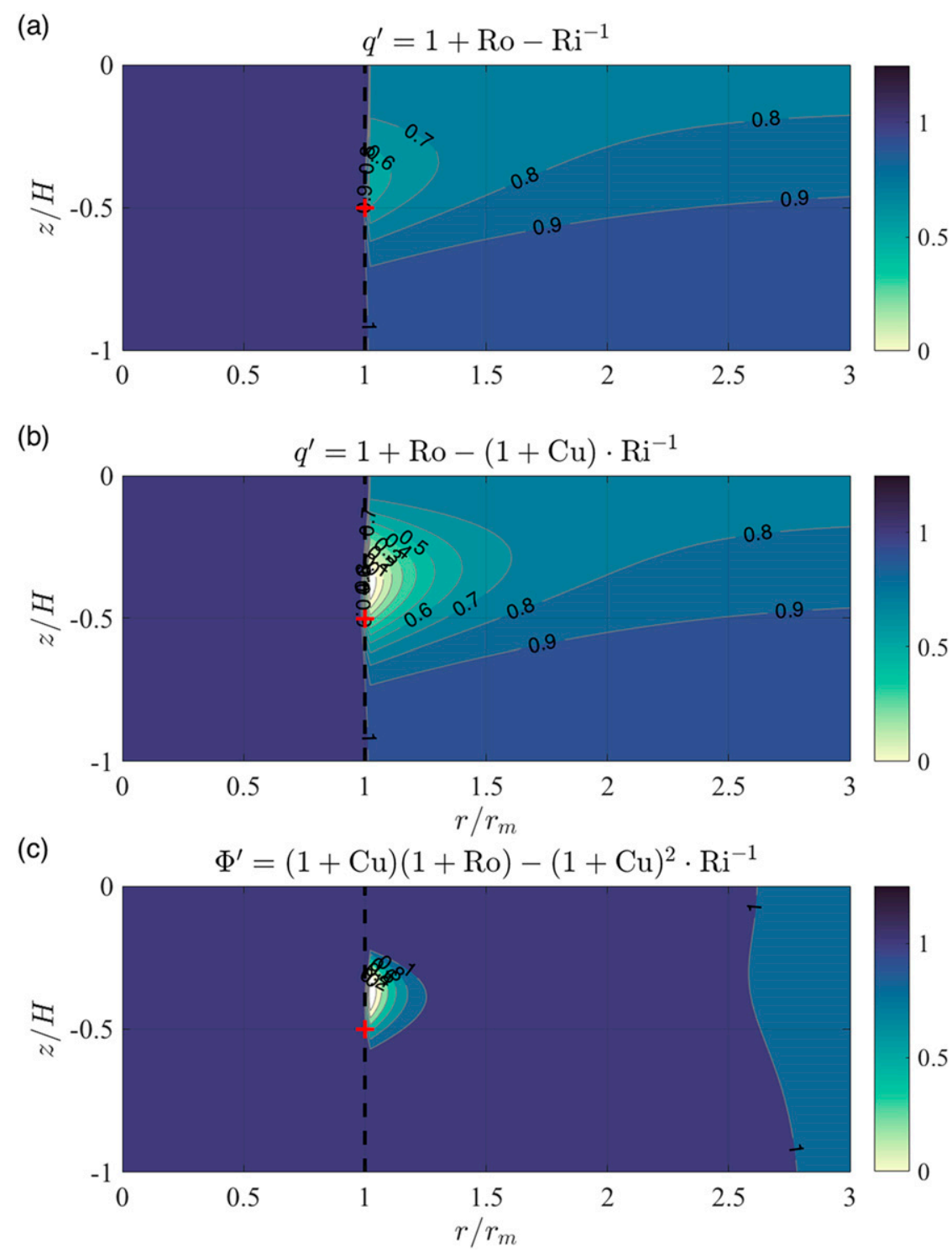

FIG. 8. As in Fig. 4, but for a cyclonic vortex.

insight into how the theoretical results of this study might translate to the observed ocean.

The Local Dynamics Experiment (LDE) eddy (Riser et al. 1986) was found within the deep thermocline. It was surveyed by a combination of ship-based expendable bathythermographs (XBTs) and Sound Fixing and Ranging (SOFAR) floats. The azimuthal velocity was estimated from float trajectories, with an average azimuthal velocity of approximately $0.25 \mathrm{~m} \mathrm{~s}^{-1}$, a radius of approximately $12 \mathrm{~km}$, and a vertical scale of $200 \mathrm{~m}$. Taking these values as $v_{m}$ and $r_{m}$, together with $f=7.3 \times$ $10^{-5} \mathrm{~s}^{-1}$ at $30^{\circ} \mathrm{N}$, one obtains a bulk Rossby number of $\mathrm{Ro}_{b}=$ $v_{m} /\left(f r_{m}\right)=-0.3$. In contrast, the vortex Rossby number, which better characterizes the core of the vortex, is approximately twice this value, $\mathrm{Ro}_{v}=2 \mathrm{Ro}_{b}=\zeta_{o} / f=-0.6$. Indeed, for Rankine vortices the core vorticity is $\mathrm{Ro}_{v}$. We have estimated a normalized vertical stratification from the stretching term in the potential vorticity of Riser et al. (1986), obtaining a value of $N / f=65$. This yields a bulk Richardson number of $\mathrm{Ri}_{b} \sim N^{2} /\left(v_{m} / h\right)^{2}=3.5$, where $h=H / 2=100 \mathrm{~m}$. These values, though bulk quantities, are within the range of nondimensional parameters examined above.

Numerous observations of small-scale coherent vortices are also found in the Arctic (e.g., D'Asaro 1988b; Timmermans et al. 2008; Zhao et al. 2014). Though it should be stressed that the baroclinic deformation radius is substantially smaller in these regions (Nurser and Bacon 2014) such that these coherent vortices are classified as "mesoscale," the momentum balance and nondimensional parameters associated with these vortices is comparable to SCVs at midlatitudes (D'Asaro 1988b). While a number of studies have highlighted the existence of small-scale coherent vortices in the Arctic (D'Asaro 1988b; Konstianoy and Belkin 1989), one particular set of observations has resulted in unparalleled statistics. 
(a)

(c)
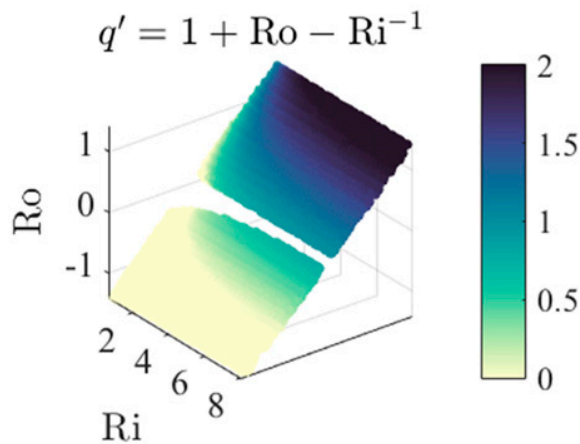

(b)

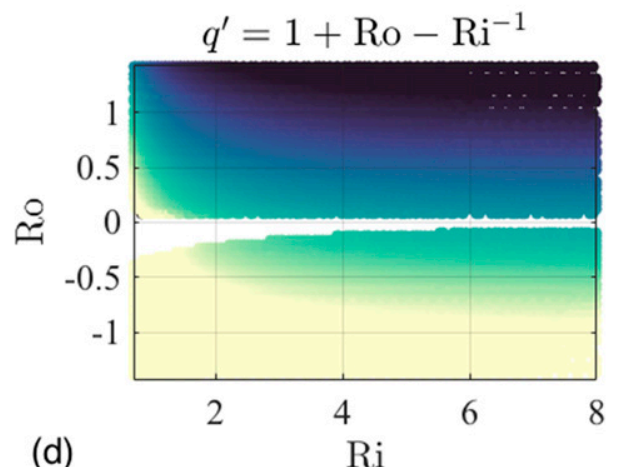

$q^{\prime}=1+\mathrm{Ro}-(1+\mathrm{Cu}) \cdot \mathrm{Ri}^{-1}$
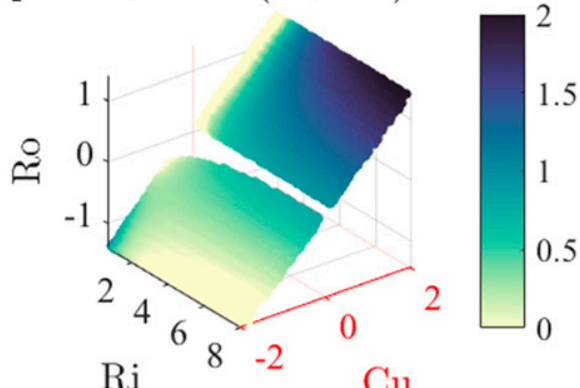

(e)

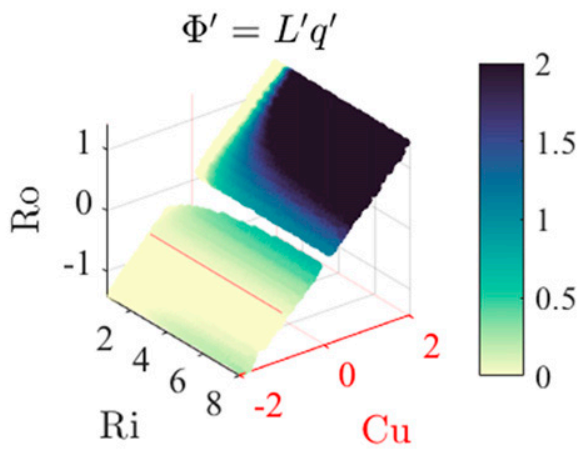

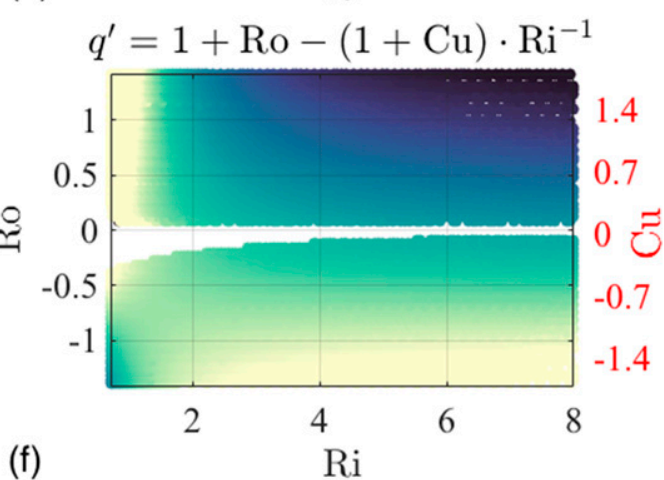

(f)

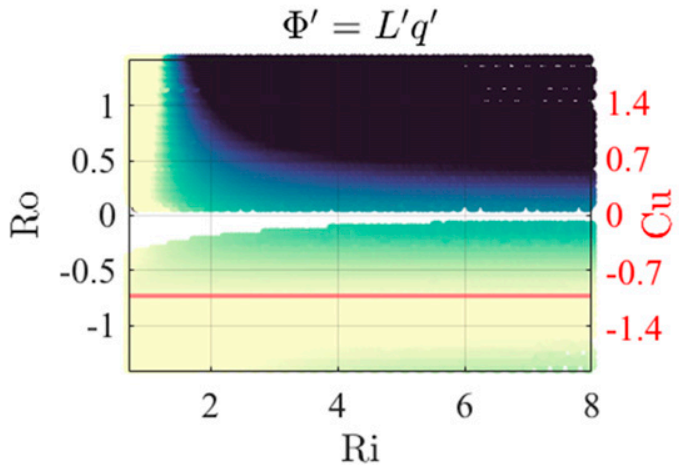

FIG. 9. Stability maps for the baroclinic front: (a),(b) nondimensional Ertel PV $q^{\prime}$, valid for straight fronts in TWB [Eq. (2)], (c),(d) $q^{\prime}$, valid for curved fronts in GWB [Eq. (9)], and (e),(f) nondimensional generalized Rayleigh discriminant $\Phi^{\prime}\left(=L^{\prime} q^{\prime}\right)$ [Eq. (8)], valid for curved axisymmetric fronts and vortices in GWB. The color map is fixed for all panels, all discriminants have been made nondimensional by dividing by $f^{2} N^{2}>0$, and the horizontal red line indicates the marginal stability curve $1+\mathrm{Cu}=0$. This parameter space is valid for the shielded Rankine vortex $(b=4.0)$ at the locations $\left(r / r_{m}, z / H\right)=(1, \pm 0.5)$ (cf. Figs. 4 and 8$)$.

Using a decade-long ice-tethered profile (ITP) record (Krishfield et al. 2008; Toole et al. 2011), Timmermans et al. (2008) and Zhao et al. (2014) surveyed a vast range of intrahalocline eddies and found that of the 127 detected eddies, only 5 of these vortices were cyclonic (Zhao et al. 2014). Moreover, the observed vortices were described as having a velocity structure comparable to the Rankine vortex while being in approximate GWB. Taking one of these vortices (ITP3-499) as a typical example, we have mapped this vortex to our nondimensional parameter space as follows. The maximum velocity is $v_{m} \approx 0.2 \mathrm{~m} \mathrm{~s}^{-1}$, radius of maximum velocity is $r_{m}=3.5 \mathrm{~km}$, nondimensional stratification is $N / f=50-80$, and the vertical scale of the vortex is $H=40 \mathrm{~m}$. We have estimated these quantities from the ITP data themselves. These values correspond to nondimensional numbers of $\mathrm{Ro}_{b}=-0.4, \mathrm{Ro}_{v}=-0.8$, and $\mathrm{Ri}_{b}=0.3-1.3$. Again, the general pattern of elevated Rossby numbers and low Richardson numbers places these observations within our regime of interest.

As a final example, we consider the SCV described by Bosse et al. (2017). Formed from convection in the Ligurian Sea, not unlike dynamics encountered in the Labrador Sea (Lilly and Rhines 2002; Legg and McWilliams 2001), the low-stratified, submesoscale vortex was characterized from hydrographic measurements. The relevant parameters estimated for the 
(a)

(c)
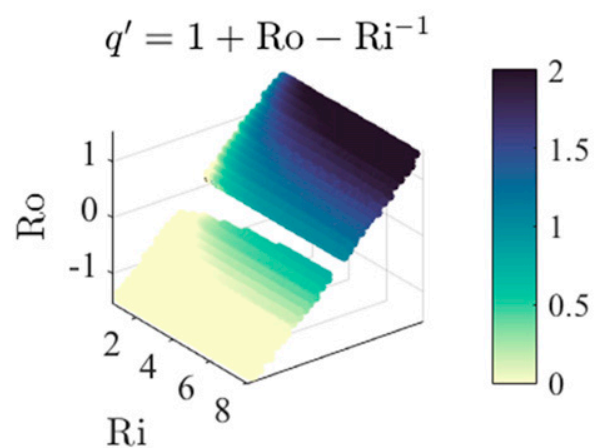

$$
q^{\prime}=1+\mathrm{Ro}-(1+\mathrm{Cu}) \cdot \mathrm{Ri}^{-1}
$$

(e)
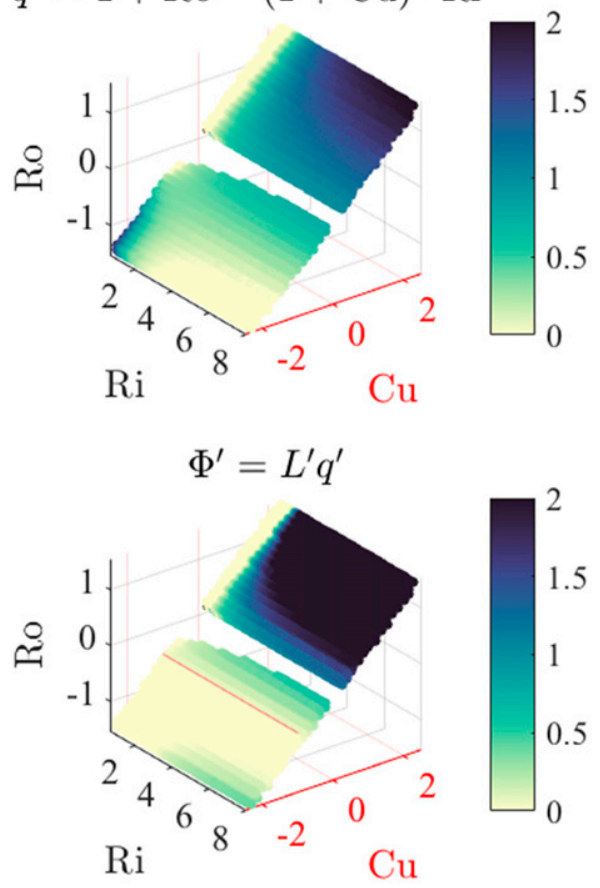

(b)

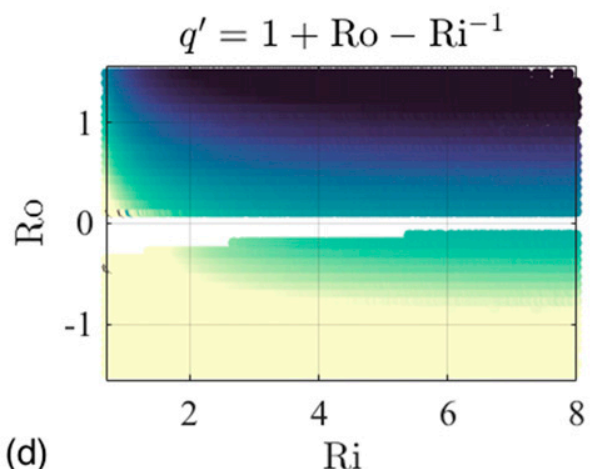

(d)

$$
q^{\prime}=1+\mathrm{Ro}-(1+\mathrm{Cu}) \cdot \mathrm{Ri}^{-1}
$$

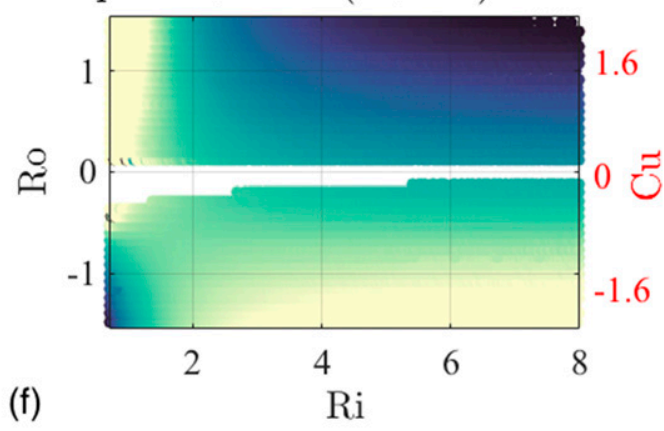

(f)

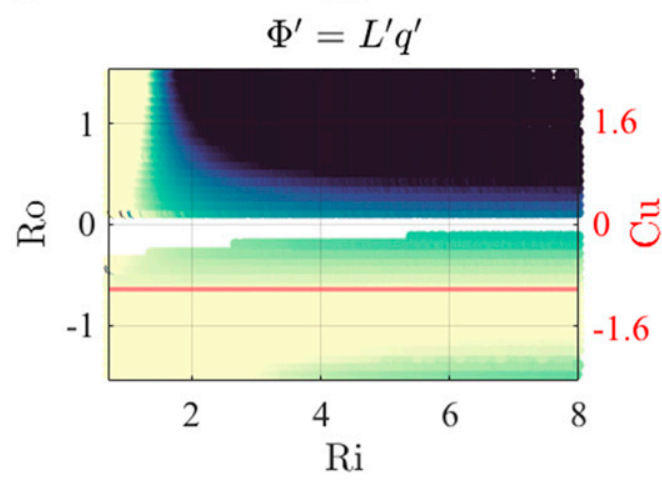

FIG. 10. As in Fig. 9, but valid for the CLM vortex ( $\alpha=1.5)$. In panels (e) and (f), note the stabilization of the flow that occurs for $\mathrm{Ri}<2$ and $\mathrm{Ro}<-1$. This corresponds to the case in which absolute angular momentum and Ertel PV are both not conserved such that $\Phi^{\prime}>0$ while $L^{\prime}<0$ and $q^{\prime}<0$. Most geophysical flows will not reside on this side of the line of marginal stability $\mathrm{Cu}=-1$.

anticyclone are $v_{m}=0.14 \mathrm{~m} \mathrm{~s}^{-1}, r_{m}=6.2 \mathrm{~km}, H=900 \mathrm{~m}$, and $N / f=4-5$ in the vortex core. Although this vertical stratification is unusually low and challenges our neglect of the meridional component of Coriolis (Colin de Verdière 2012; Kloosterziel et al. 2017; Zeitlin 2018), it is nonetheless consistent with the formation of submesoscale vortices by deep convection. The corresponding nondimensional numbers are $\mathrm{Ro}_{b}=0.23, \mathrm{Ro}_{v}=$ 0.46 , and $\mathrm{Ri}_{b}=1.65-2.6$.

\section{b. Comparison with stability diagrams}

Other SCV observations exist (e.g., Lilly and Rhines 2002; Paillet et al. 2002; Meunier et al. 2018). They are almost universally anticyclonic, characterized by elevated relative vorticity magnitudes $\left(\left|\mathrm{Ro}_{b}\right|>0.1\right)$ relative to those found in QG flows, and have horizontal scales on the order of the deformation radius. We summarize a number of these characteristics in Table 1 for the aforementioned vortices and overlay $\mathrm{Ro}_{b}$ and $\mathrm{Ri}_{b}$ on stability maps shown in Fig. 13. For comparison with the LDE eddy, we include a vortex found in realistic numerical simulations (Gula et al. 2019) and thought to reflect dynamics associated with the LDE eddy. Though qualitative, these comparisons prove insightful and support our proposed hypothesis.

Note that the nondimensional Rayleigh criterion, $\Phi^{\prime}<0$, describes the set of observed and simulated vortices well, as stable regions $\left(\Phi^{\prime}>0\right)$ include most vortices (Figs. 13b,d). An exception is the Arctic halocline vortex (Timmermans et al. 2008), which is 
(a)

(c)
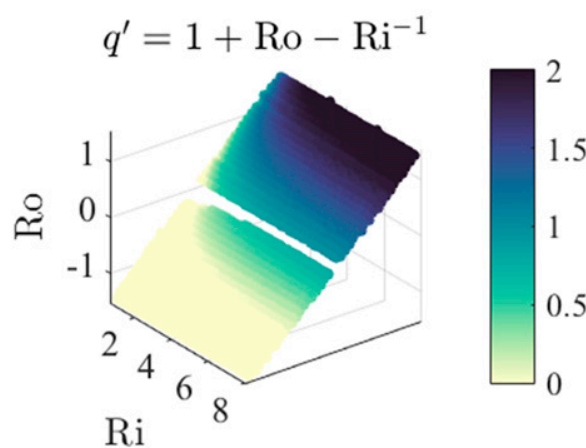

(e)

$$
q^{\prime}=1+\mathrm{Ro}-(1+\mathrm{Cu}) \cdot \mathrm{Ri}^{-1}
$$
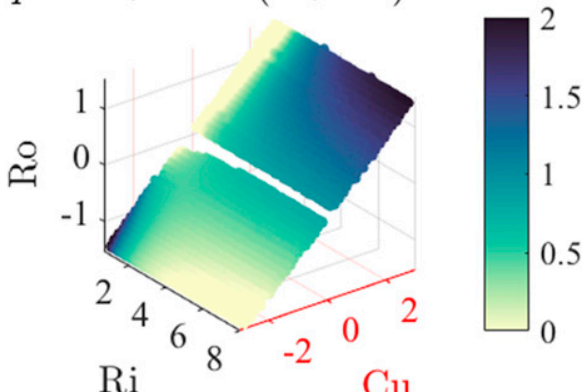

(d)

(b)
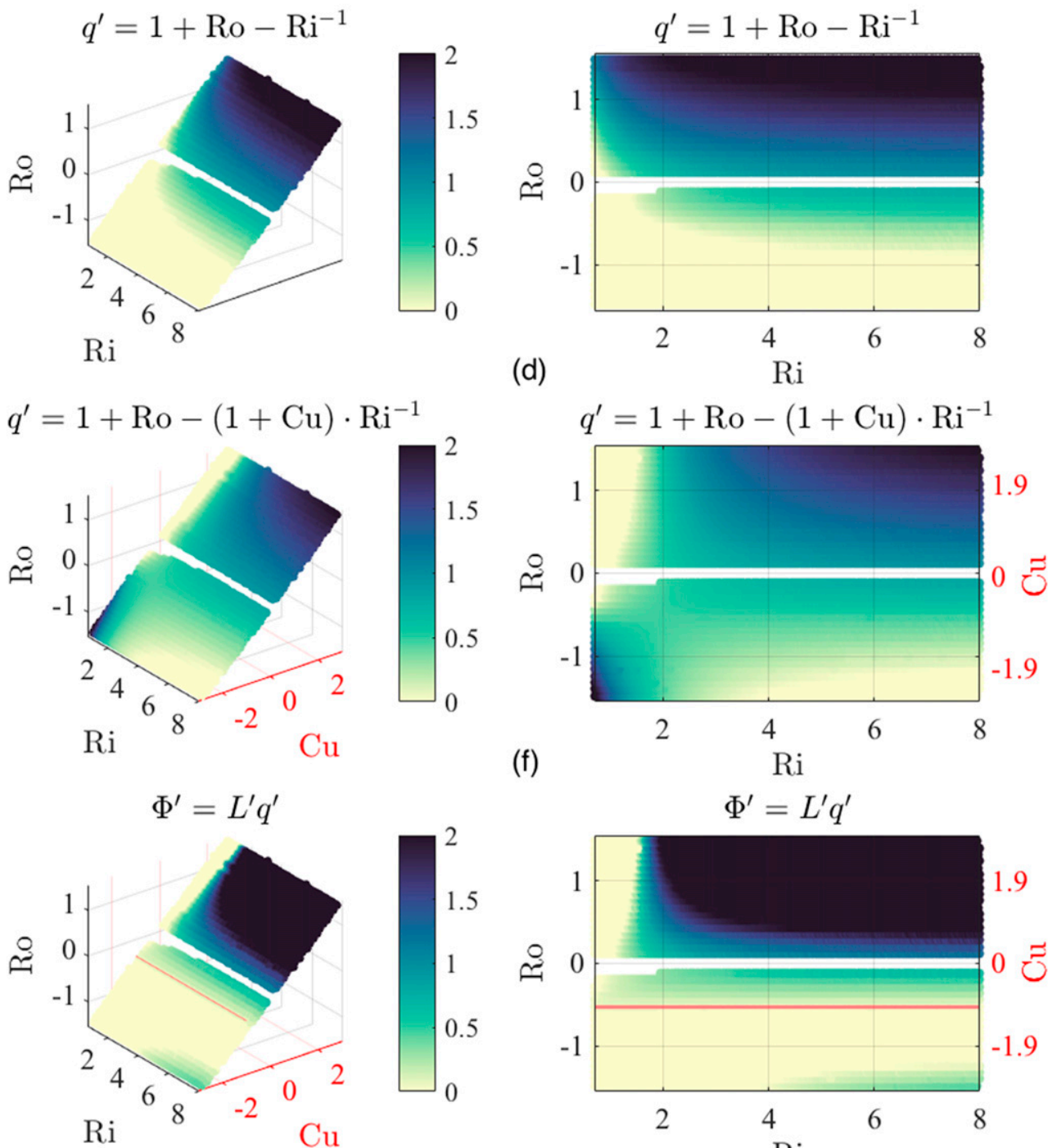
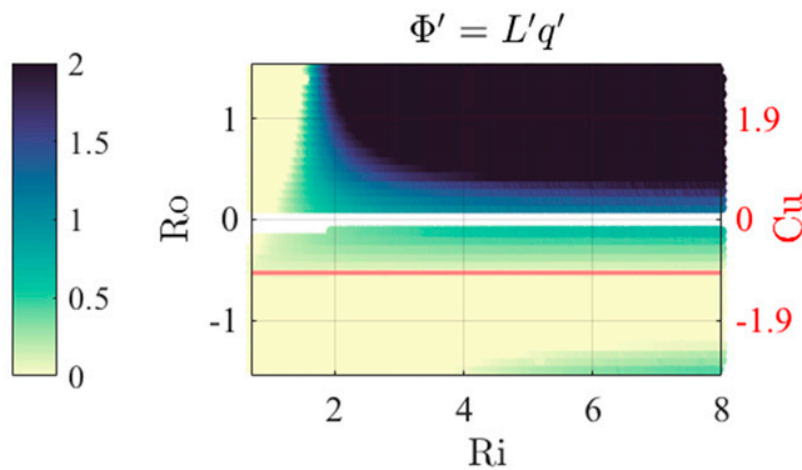

FIG. 11. As in Fig. 9, but valid for the CLM vortex $(\alpha=2.0)$. This case is equivalent to the Gaussian vortex. This model is thought to accurately reflect less intense vortices or older vortices that may have traveled far from their point of origin (Paillet et al. 2002).

found outside of the stable region in the global average $\left.\left(\left\langle\Phi^{\prime}\right\rangle\right\rangle 0\right)$. This might be due, for example, to (i) uncertainty in the estimated Richardson number or (ii) frictional effects with the ice that are not incorporated into $\Phi^{\prime}$. In summary, while some scatter is expected, this qualitative agreement supports use of the generalized Rayleigh criterion. Second, the histograms of stable Ro (Figs. 13c,e) are maximum near the vorticity values of the observations. Given that observed, submesoscale vortices universally form in low-stratified environments (i.e., boundary layers) and proceed via advection and subduction into the ocean interior but which is also characterized by low vertical stratification, these qualitative comparisons suggest the asymmetry in the distribution of coherent vortices (McWilliams 1985; Bane et al. 1989; Konstianoy and Belkin 1989; McWilliams 2016) might originate in the boundary layer and be explained by the vortex tilting arguments discussed in Part I. This motivates the following hypothesis for the generation and evolution of smallscale, coherent vortices in the ocean interior.

\section{c. Generation and evolution of small-scale, coherent vortices}

Coherent vortices, such as SCVs and polar mesoscale vortices, typically are generated as a result of cyclogeostrophic adjustment of near-boundary layer fluid. The mechanisms thought to create such phenomena include (i) baroclinic instability of fronts (Spall 1995; Boccaletti et al. 2007; Timmermans et al. 2008; Wenegrat et al. 2018), (ii) frictionally induced forcing of fluid parcels 
(a)

(c)
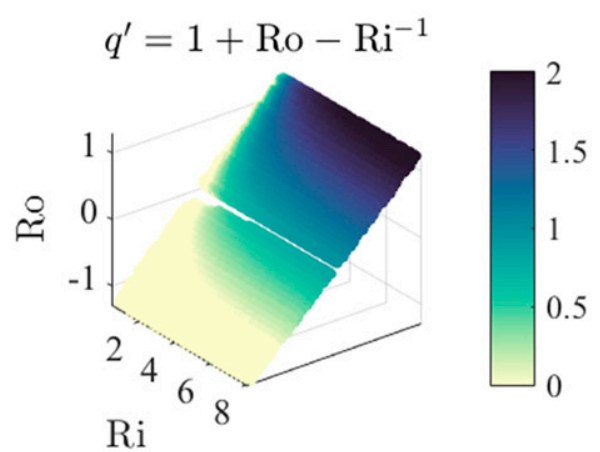

$$
q^{\prime}=1+\mathrm{Ro}-(1+\mathrm{Cu}) \cdot \mathrm{Ri}^{-1}
$$

(e)
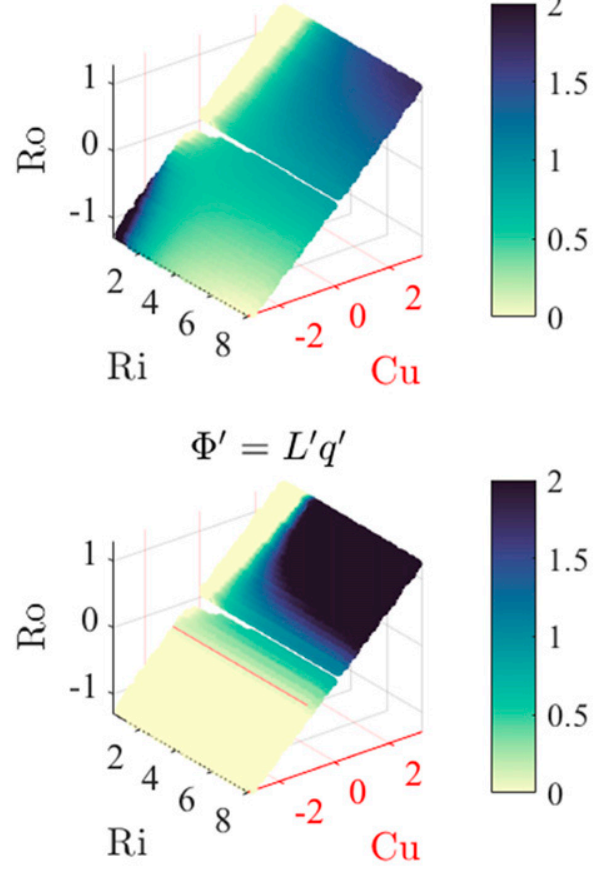

(b)

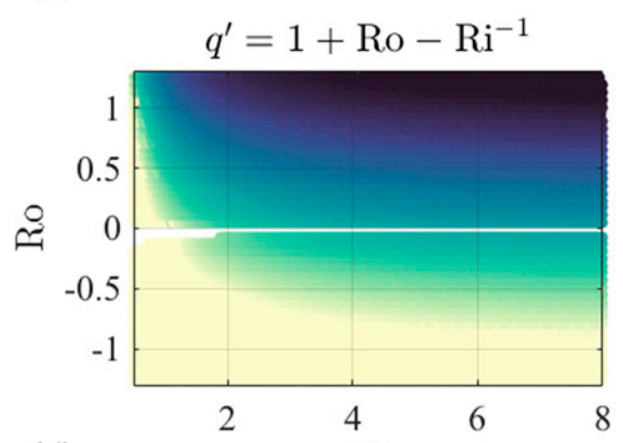

(d)

$\mathrm{Ri}$

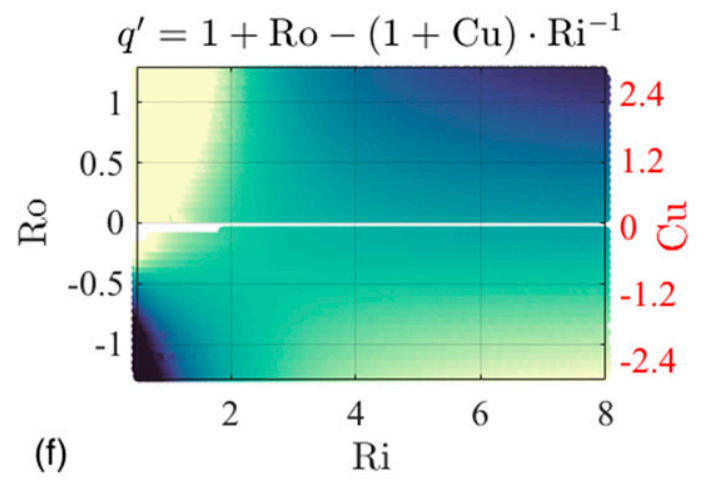

$(f)$

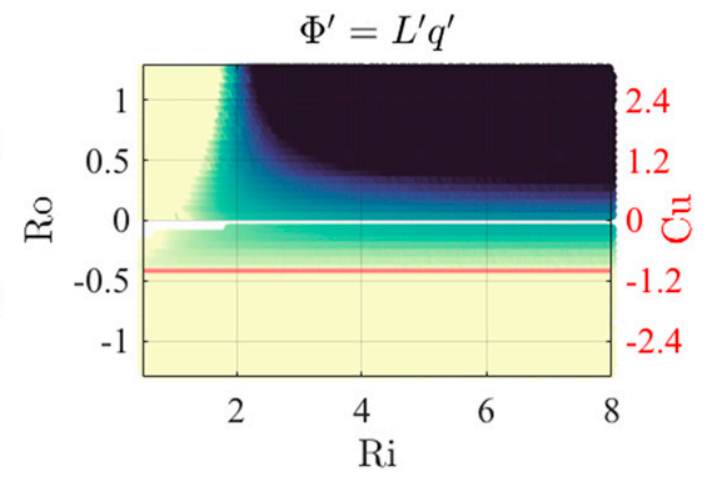

FIG. 12. As in Fig. 9, but valid for the CLM vortex $(\alpha=2.5)$. This model is thought to most accurately describe intense vortices, such as submesoscale coherent vortices.

(D'Asaro 1988a; Thomas 2008; Jiao and Dewar 2015; Gula et al. 2016; Brannigan et al. 2017; Perfect et al. 2018), and (iii) buoyancy-induced forcing of fluid parcels (Helfrich and Battisti 1991; D'Asaro et al. 1994; Legg and McWilliams 2001; Hogan and Hurlburt 2006; Deremble 2016; Gordon et al. 2017; Garabato et al. 2017; Meunier et al. 2018). Note that these boundary layers including ocean surface, bottom, and ice-ocean boundary layers. Because such boundary waters have reduced stratification, $N / f \ll 100$, if fluid is trapped within the cores of the vortices, this low stratification can persist even when the vortices exit these boundary layers.

To conserve both absolute angular momentum and Ertel $\mathrm{PV}$, the vortex will alter its nondimensional numbers-that is, to keep their product $\Phi^{\prime}=L^{\prime} q^{\prime}$ constant. Owing to reduced stratification, the cyclonic vortex cannot appreciably alter in Ri but will reduce in relative vorticity, $\mathrm{Ro}=\bar{\zeta} / f$, while the anticyclonic vortex will remain relatively unchanged. If, however, the cyclonic vortex is unable to alter its vorticity (e.g., it is rotating too quickly), it will lose kinetic energy in the form of dissipation due to symmetric instability and, eventually, Kelvin-Helmholtz instability. For the unstable cyclone, this will occur quickly, with growth rates scaling as $\sigma^{2} \sim-f^{2} \Phi^{\prime}$ (section e of appendix A in Part I). For the anticyclone, being weakly stable/unstable, this decay will require significantly more inertial periods. The decay time scale of the cyclone under symmetric instability can be estimated as $\tau=2 \pi / \sigma \approx T_{i} /\left(-\Phi^{\prime}\right)^{1 / 2}$, where $T_{i}=2 \pi / f$ is the inertial period. In summary, it is conservation of $\Phi^{\prime}$ in the presence of low $N^{2}$ that shapes 


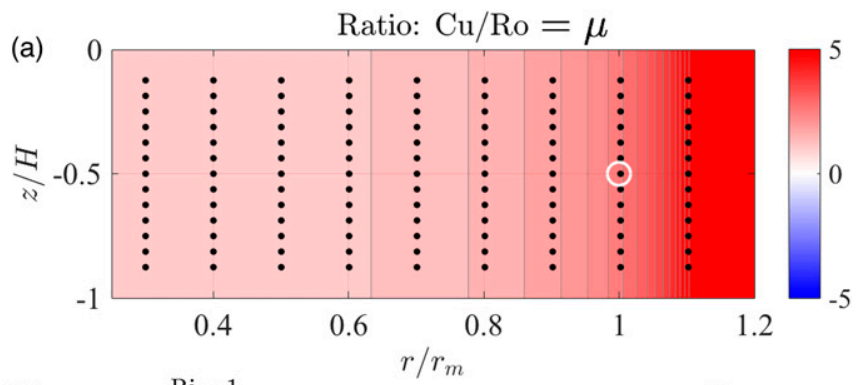

\section{List of Vortices}

+ Riser et al. (1986)

Paillet et al. (2002)

$\triangle$ Timmermans et al. (2008)

$\bigcirc$ Bosse et al. (2017)

$\times$ Meunier et al. (2018)

+ Gula et al. (2019)

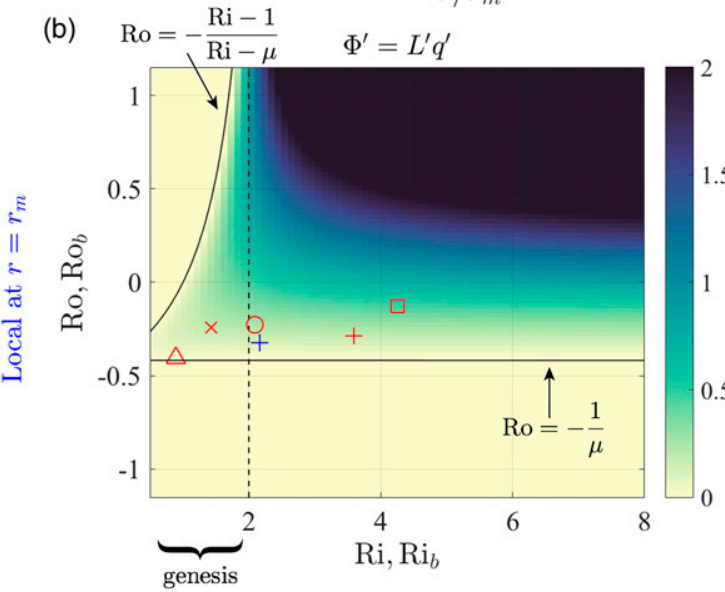

(c) Probability of Observing $|\mathrm{Ro}|$ for $\mathrm{Ri}<2$

(d)
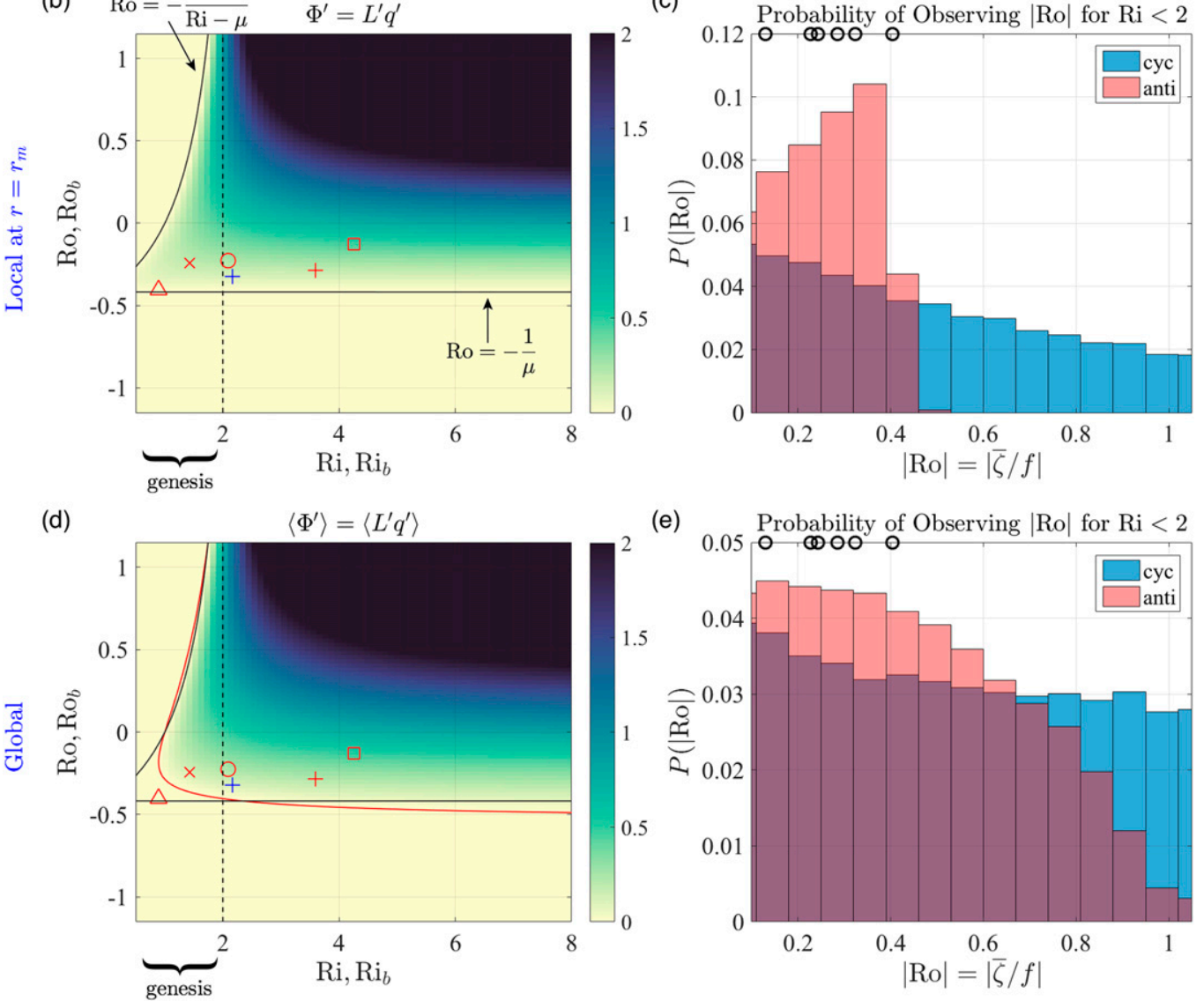

(e) Probability of Observing $|\mathrm{Ro}|$ for $\mathrm{Ri}<2$

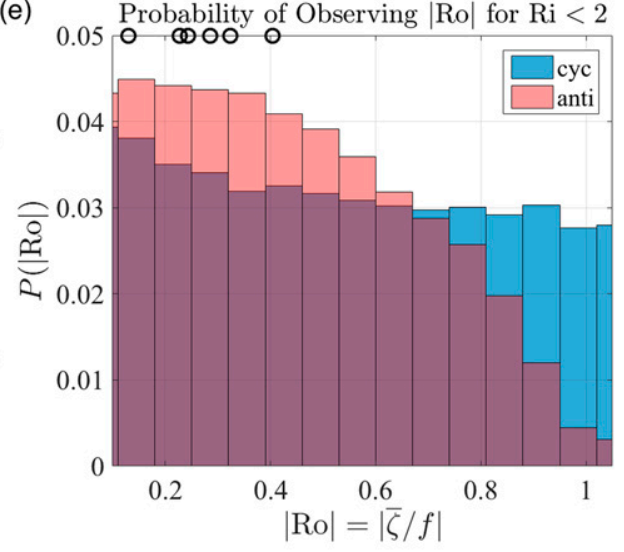

FIG. 13. (a) Curvature-vorticity ratio $\mu=\mathrm{Cu} / \mathrm{Ro}$, for the CLM vortex $(\alpha=2.5)$, and valid for both cyclones and anticyclones. The white circle highlights $\mathrm{Cu} / \mathrm{Ro}$ at the location, $r / r_{m}=1$ and $z / H= \pm 0.5$, and black dots highlight locations entering into the global average. (b) Stability discriminant $\Phi^{\prime}$, valid at $r / r_{m}$ (cf. Fig. 12f). (c) Probability of a stable occurrence as a function of $|\mathrm{Ro}|$ for $\mathrm{Ri}<2.0$, for anticyclones (red) and cyclones (blue). Black circles at the top of the plot indicate observed $\mathrm{Ro}_{b}$. (d) Global average $\left\langle\Phi^{\prime}\right\rangle$, obtained from averaging $\Phi^{\prime}$ at the locations (black dots) in (a). (e) As in (c), but obtained from stable states at locations (black dots) in (a). In (b) and (d), we depict $\mathrm{Ro}_{b}$ and $\mathrm{Ri}_{b}$ of observed (red) and simulated (blue) vortices (cf. Table 1). To delineate stable and unstable regimes, we also overlay in (b) and (d) the local (solid black) and global (solid red) marginal stability curves. Our interpretation is that recently formed vortices reside on the left in (b) and (d) (i.e., low Ri) and slowly drift to the right with increasing age (i.e., elevated Ri).

the distribution of relative vorticity at these small horizontal scales.

\section{d. Cyclonic SCVs}

While submesoscale cyclones are found in the ocean interior, observations are rare and are more frequent near coasts.
A great example is the vortex recently documented by de Marez et al. (2020). Observed in the Gulf of Aden, it is believed to have been generated through barotropic shear instability as dense waters exited the Arabian Sea. As determined from coincident SeaSoar and acoustic Doppler current profiler (ADCP) measurements, it has the following characteristics: 


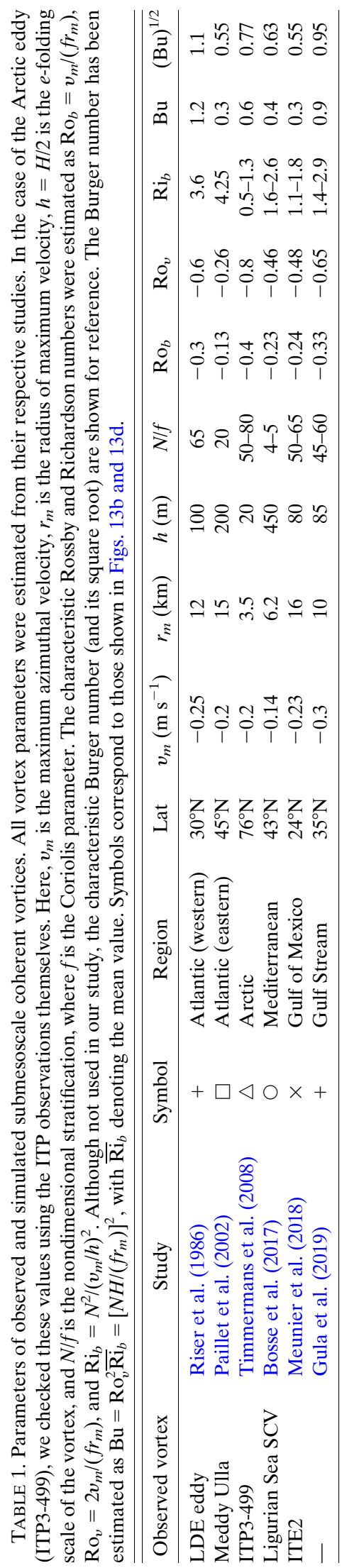

$v_{m}=0.5 \mathrm{~m} \mathrm{~s}^{-1}, r_{m}=16 \mathrm{~km}, h=H / 2=200 \mathrm{~m}$, and $N / f=150-$ 200 , yielding $\mathrm{Ro}_{b}=1.0, \mathrm{Ro}_{v}=2.0$, and $\mathrm{Ri}_{b}=3.5-6.2$. We mention this for context, noting that it has been observed immediately following generation. It will therefore be interesting to know whether this vortex is symmetrically unstable, helping to support the mechanisms thought to shape the distribution of relative vorticity.

\section{Discussion}

The ability of intense anticyclones to persist in stratified flows has been noted for some time. Lazar et al. (2013), for example, derived an instability criterion valid for barotropic vortices embedded in a vertically stratified environment. One of their main findings was that increased stratification and turbulent eddy viscosity tended to stabilize a vortex, explaining the stability of anticyclonic vortices with order-1 Rossby numbers. While we, too, find the possibility of stable anticyclones at high Rossby numbers (although this is muted somewhat by the constraint $L^{\prime}=1+\mathrm{Cu}>0$ ), we suggest here that it is the pronounced curvature in the presence of low stratification that creates this stability. Curvature modifies the baroclinic structure and, in an effort to conserve $\Phi^{\prime}$, this in turn modifies gradient Rossby and Richardson numbers permitted for stability. Examined from another perspective, one finds that the discriminant $\Phi^{\prime}$ at fixed gradient Rossby and Richardson numbers increases in magnitude. Moreover, this increase in stability occurs in the absence of viscosity, suggesting stronger vortices are possible when accounting for viscous terms.

This is consistent with a recent study by Yim et al. (2019), who extended the work of Lazar et al. (2013) to the continuously stratified case. While their focus has been on explaining the existence of surface-intensified vortices, their results nevertheless appear complementary to this study. In particular, in the presence of absolute angular momentum conservation, we are unable to rationalize stable anticyclones with $|\mathrm{Ro}|>0.5$ (cf. Fig. 13d). (An exception is the unique case in which both $L$ and $q$ are not conserved.) This constraint on gradient Rossby numbers corresponds to a limit on the curvature number, $\mathrm{Cu}>-1$. For anticyclones, this implies that the centripetal acceleration can never be larger than the magnitude of the Coriolis acceleration divided by two, causing Gaussian vortices, for example, to have $\operatorname{Ro}_{b}>-0.5$ (Mahdinia et al. 2017). ${ }^{7}$ This constraint can also be inferred from the quadratic expression used to simulate or estimate velocities for flows in GWB (Penven et al. 2014). Thus, unless the axisymmetric constraint is relaxed (Billant and Gallaire 2005), the existence of anticyclones with Rossby numbers near one must invoke a physical mechanism not present in $\Phi<0$. Viscosity is a reasonable candidate.

Mahdinia et al. (2017) investigated the linear stability of three-dimensional Gaussian vortices in rotating, stratified flow using numerical methods. Their dynamical regime of interest

\footnotetext{
${ }^{7}$ This can be rationalized by noting that $\mathrm{Cu}>-1$ implies $\mathrm{Ro}_{b} \sim\left(v_{m}^{2} / r_{m}\right) /\left(f v_{m}\right)=v_{m} /\left(f r_{m}\right)>-1 / 2$.
} 
was centered on Rossby numbers between \pm 0.5 and Burger numbers $^{8}$ of $0.02-2.3$. In rough terms, these nondimensional numbers correspond to bulk Richardson numbers of 0.3-3.0. Their simulated vortices were embedded in a background stratification of $N / f=10$. Mahdinia et al. (2017) found that of the 130 simulated Gaussian vortices, only 4 (all cyclones) were neutrally stable. Moreover, this occurred within a small dynamical regime: $\mathrm{Ro}_{b}=0.02-0.05$ and $\mathrm{Bu}=0.85-0.95$. Also notable, the authors found that unstable anticyclones generally had slower growth rates when compared with the growth rates of cyclones, and that this slow-growth area occupied a large portion of the parameter space. Anticyclones tended to become more stable with increasing $\mid$ Ro $\mid$ and cyclones decreased in stability (increased growth rate) with increasing $|\mathrm{Ro}|$.

Though a direct comparison with our results is somewhat limited by the use of gradient Richardson numbers rather than bulk quantities, e.g., Burger numbers, the destabilization of cyclones and stabilization of anticyclones with increasing |Ro| is qualitatively consistent with our results, as in some cases increased $\mathrm{Cu}$ for anticyclones results in stabilization of the flow (cf. Fig. 4c). Also, the conclusion that anticyclones should persist for longer periods than cyclones in low-stratified waters is consistent with the present study. A final similarity is evident in Fig. 13d of our study. Mahdinia et al. (2017) document weakly unstable anticyclones for nearly all Ro. Similarly, as we began to examine an average of $\Phi^{\prime}$ within the whole of the vortex [cf. section $2 b(4)$ ], we noted that $\left\langle\Phi^{\prime}\right\rangle$ can become slightly negative for anticyclones when $\mathrm{Ri}<2$. To the extent that the growth rate scales with $\Phi^{\prime}$, this is consistent with the observation of weakly unstable anticyclones in lowstratified waters.

The final study with which we compare our findings is a field campaign in the vicinity of the Gulf Stream. Shcherbina et al. (2013) documented an observational effort to characterize upper-ocean dynamics at small horizontal scales (i.e., submesoscales) in this region. The authors employed current measurements from two ships moving in parallel, and estimated gradients in velocity between ships and in the direction of motion, characterizing the upper-ocean velocity field in terms of vorticity, divergence and strain rate. In examining the joint probability distribution function of vorticity $\zeta$ and strain rate $\alpha$ in both observations and high-resolution numerical simulations of the upper ocean, Shcherbina et al. (2013) demonstrated three interesting characteristics. In this context, $\alpha=\left(S_{n}^{2}+S_{s}^{2}\right)^{1 / 2}$, where $S_{n}$ and $S_{s}$ are the normal and shear components of strain rate (e.g., Isern-Fontanet et al. 2004). First, cyclonic vorticity in excess of $|\mathrm{Ro}|=1.0$ was typically associated with large strain rates, and approached a pure shear relationship, $\alpha=|\zeta|$, for large Ro. This suggests large cyclonic flow is predominantly found within upper-ocean fronts (i.e., where curvature is small). Second, anticyclonic flows had limited $|\mathrm{Ro}|$ (values smaller than 1.0 being most

\footnotetext{
${ }^{8}$ The Burger number is defined as the squared ratio of the deformation radius to the radial scale of the vortex, $\mathrm{Bu}=\left(r_{d} / r_{m}\right)^{2}=\left[N H /\left(f r_{m}\right)\right]^{2}$, and can roughly be related to bulk Rossby and Richardson numbers as follows: $\mathrm{Bu}=\left[N H /\left(f r_{m}\right)\right]^{2}=\left[2 v_{m} /\left(f r_{m}^{2}\right)\right]^{2}\left[N^{2} /\left(v_{m} / h\right)^{2}\right]=\mathrm{Ro}_{v}^{2} \mathrm{Ri}_{b}$.
}

common). Third, the probability of $|\zeta| \gg \alpha$ (indicative of solidbody rotation) was much higher for anticyclones than for cyclones, suggesting anticyclonic flows are preferentially found within coherent "eddy-like structures" (Shcherbina et al. 2013). Last, we mention that these observationally motivated findings are in good agreement with conclusions of Roullet and Klein (2010), who examined the distribution of relative vorticity in a surface-forced, $x$-periodic primitive equation model.

The above distinction between vorticity skewness created by fronts and vorticity skewness created by coherent vortices is an important one and is consistent with the present study. For fronts in TWB (i.e., $\mathrm{Cu}=0$ ), Eq. (8) states that, for stable flow, $\mathrm{Ro}>-1+\mathrm{Ri}^{-1}$ [cf. Eq. (2)]. Thus, one expects vorticity skewness to result from elevated cyclonic flow and bounded anticyclonic flow. For fronts in GWB (i.e., $|\mathrm{Cu}|>0$ ), Eq. (8) states that, for stable flow, $\Phi^{\prime}=(1+\mathrm{Cu})(1+\mathrm{Ro})-(1+$ $\mathrm{Cu})^{2} \mathrm{Ri}^{-1}>0$. While this expression is not as easily manipulated to reveal skewness in the distribution of Ro (see our paragraph below), one can make heuristic progress by examining $q^{\prime}$ and $L^{\prime}$ separately. Recall, Eq. (9) predicts a decrease in $\mathrm{PV}$ for cyclones and an increase in PV for anticyclones, relative to the TWB case. Also, recall that $0<L^{\prime}<1$ for inertially stable anticyclones and $L^{\prime}>1$ for inertially stable cyclones. Therefore, $\Phi^{\prime}=L^{\prime} q^{\prime}$ is a muted version of $q^{\prime}$ for anticyclones and $\Phi^{\prime}$ is an amplified version of $q^{\prime}$ for cyclones. The final result is that anticyclones are weakly stabilized at low $\mathrm{Ri}$, while cyclones are strongly destabilized.

Firmer statements can be made using marginal stability curves obtained in section $2 b(5)$. Solving for the roots of $\Phi^{\prime}=0$ and assuming $\mu>1$, one concludes that for low Richardson numbers, $\mathrm{Ri}<\mu$, all cyclonic curved fronts are unstable so long as $\mathrm{Ro}>\mathrm{Ro}_{1}$, where $\mathrm{Ro}_{1}=(1-\mathrm{Ri}) /(\mathrm{Ri}-\mu)$ has been labeled the "baroclinic" root. In contrast, anticyclonic flows can be stable for these same gradient Richardson numbers. This result is nontrivial since it is traditionally assumed that cyclonic flows are more stable than anticyclonic flows (Hoskins 1974). ${ }^{9}$ Assuming $\mu>1$ and substituting in $\mathrm{Ri} \leq 1$, for example, we find that all cyclonic fronts are unstable for Richardson numbers less than unity, while anticyclonic fronts and vortices can be weakly or marginally stable for moderate magnitude Rossby numbers, $0<|\mathrm{Ro}|<\left|\mu^{-1}\right|$. The skewness in the distribution of Ro can therefore be explained by this expression for low Richardson numbers, $\mathrm{Ri}<\mu$, and for moderately large curvature-vorticity ratios.

In summary, the nondimensional criterion examined in this study, $\Phi^{\prime}=L^{\prime} q^{\prime}<0$, explains both 1) the classical finding of a dominance of cyclonic vorticity (positive vorticity skewness)

\footnotetext{
${ }^{9}$ Hoskins writes, "However, in frontal regions, the Richardson number can be less than unity without symmetric instability being possible" (Hoskins 1974, p. 481). Here, Hoskins (1974) is speaking about cyclonic fronts in TWB and the influence of such relative vorticity on stability. However, for these same gradient Richardson numbers and for fronts in GWB, we find in this study that curvature modifies this statement: no longer is the cyclonic front stable, but rather it is the anticyclonic (curved) front that can become weakly stable for $\mathrm{Ri}<1$.
} 
for straight fronts (Rudnick 2001; Shcherbina et al. 2013; Buckingham et al. 2016) and 2) the unexplained dominance of anticyclonic vorticity (negative vorticity skewness) for eddying flows (Shcherbina et al. 2013; Roullet and Klein 2010). Perhaps the reason this negative skewness in the distribution of Ro has not been explained before is that the generalized Rayleigh criterion needs to be expressed in terms of gradient Richardson numbers, something that is not traditionally done. This naturally follows from a "frontal" perspective of the criterion presented in section 2c of Part I.

\section{Summary, implications, and future steps}

In this portion our study, we have investigated the outcome of the criterion $\Phi^{\prime}=L^{\prime} q^{\prime}<0$ [cf. Eq. (8)] applied to curved density fronts. In particular, we have focused on the dynamical regime in which symmetric instability is expected to occur-that is, Richardson numbers near 1 (Stone 1966, 1970). While several outcomes of the criterion could be mentioned, the principal finding is that curvature in lowRichardson-number flows can act to destabilize cyclonic fronts and stabilize anticyclonic fronts to symmetric instability, a feature that increases with increasing curvature magnitude, $|\mathrm{Cu}|$. Although this possibility was noted in Part I, its existence for realistic values of Ro and $\mathrm{Ri}$ (or $N / f$ ) could not have been predicted without first examining base flows with known curvature-vorticity relationships, $\mu=\mathrm{Cu} / \mathrm{Ro}$. This is therefore a new and significant result. ${ }^{10}$

While discussions in this study have largely been framed in terms of vortices, our results are additionally applicable to curved fronts if one confines such a discussion to the region $r<r_{m}$. In reference to the meandering front schematic given in Fig. 2 of Part I, this would suggest that the blue-shaded region is susceptible to symmetric-inertial instabilities, while the redshaded region is marginally stable. Given the potential for cyclonic curved flows to be more susceptible to symmetric instability than anticyclonic flows, this implies climate-relevant dynamics might be occurring within meandering curved fronts that may not presently be accounted for. For example, there may be enhanced tracer exchange (e.g., Thomas and Taylor 2010; Taylor and Ferrari 2010; Smith et al. 2016) occurring within cyclonic meanders but not anticyclonic meanders of fronts. This may require further study, however, since stretching terms in $\Phi^{\prime}$ might adjust more readily within a meandering front than is possible within a vortex. With respect to a recent study of tracer exchange at the northern "wall" of the Gulf Stream (Wenegrat et al. 2020), this study took place along a portion of the front that was relatively straight; we anticipate curvature effects are therefore minimal.

The development of parameterizations of submesoscale processes for use within coarser-resolution ocean models is ongoing, with the tremendous foresight that these processes

\footnotetext{
${ }^{10}$ The skewness in the distribution of relative vorticity could have been predicted by examining the bound on Rossby numbers imposed by the baroclinic root $\mathrm{Ro}_{1}$. However, since this itself depends upon $\mu$, one must explore realistic base flows.
}

might be important for large-scale ocean dynamics, influencing, for example, energy, buoyancy, and tracer budgets (Boccaletti et al. 2007; Thomas and Taylor 2010; Fox-Kemper et al. 2011; Smith et al. 2016; Bachman et al. 2017). In an effort to make progress, oceanographers typically approximate these processes as occurring at fronts that are in TWB, as mentioned in the introduction to Part I. However, this study suggests a valid question to ask is, "At horizontal scales for which symmetric instability takes place, to what extent can frontal curvature be neglected?" Indeed, this study would suggest that for submesoscale fronts, or those fronts at which Rossby and Richardson numbers approach 1.0, it may be necessary to consider frontal curvature. Moreover, even at mesoscales, this dynamic could be relevant. The relevant nondimensional quantity is the curvature number, $\mathrm{Cu}=2 \bar{v} /(f r)$. In summary, if frontal stability is modified by curvature, then curvature dynamics may be relevant to the aforementioned budgets. This includes bolus transport of nutrient-rich and rare, tracer-rich waters via coherent vortices, as well as enhanced exchange between the deep and upper ocean, thereby affecting oceanatmosphere exchanges. Submesoscale-resolving ocean simulations and observations are therefore necessary to explore these hypotheses and to represent such processes within coarser-resolution ocean models.

The assumptions made in obtaining the instability criterion $\Phi<0$ restrict its application to inviscid flow on the $f$ plane. In addition, the fronts are assumed to be far enough from the equator that the meridional component of Coriolis is negligible. Thus, to consider its application to a greater range of frontal types and locations, for example, tropical instability waves (Marchesiello et al. 2011; Holmes et al. 2014), it may be necessary to generalize the governing equations and associated instability criterion by 1) permitting displacements of fluid parcels over larger meridional scales such that $f$ can vary, perhaps using spherical coordinates, and 2) including the meridional component of Coriolis in the governing equations.

The overwhelming majority of submesoscale vortices observed in the oceans are reported as being anticyclonic (McWilliams 1985). This has been reiterated more recently (McWilliams 2016), suggesting that a fundamental understanding of the dynamics is lacking. Here, we suggest that, while the stability criterion examined in our study may not completely explain the dominance of anticyclonic versus cyclonic curvature, it is clear that Eq. (8) applies and may help to explain the observed asymmetry in the distribution of smallscale, coherent vortices in the ocean interior. It is important to stress that formation mechanisms themselves can favor vorticity of a certain sign (D'Asaro 1988a; Gula et al. 2016) and that viscous effects can be important (Lazar et al. 2013; Yim et al. 2019). Nevertheless, given the potential for anticyclones to persist for longer lifetimes and cyclones to dissipate more rapidly, this study has relevance for energy, momentum, and biogeochemical tracer fluxes in the oceans, with corresponding challenges for representation within Earth system models.

Acknowledgments. This study was made possible by a Marie Skłodowska-Curie Actions (MSCA) Individual Fellowship from the European Commission (Proposal 798319). The contributions 
of the authors to the published work are as follows. Author Buckingham conceived of the idea, devised and executed the study, and wrote the paper. Author Gula provided early model support for these ideas. Author Carton helped to ensure that the mathematics were correct when applying the criterion to balanced flows. We thank the editor and two reviewers for their support of the paper. Buckingham thanks G. Crystle (UBO) for encouragement and initial inspiration, G. Roullet (UBO) for earlier discussions about vorticity skewness, and C. De Marez (UBO) for valuable input on an early draft. We again acknowledge formative comments made by S. Griffies (NOAA/GFDL) and G. Nurser (NOCS), as well as recent conversations with the following individuals: P. Penven (IRD), N. Lahaye (Ifremer/Inria), C. Menesguen (Ifermer), J. Paillet (Ifremer), M-L. Timmermans (Yale), and T. Meunier (WHOI).

\section{REFERENCES}

Adams, K. A., P. Hosegood, J. R. Taylor, J.-B. Sallée, S. Bachman, R. Torres, and M. Stamper, 2017: Frontal circulation and submesoscale variability during the formation of a Southern Ocean mesoscale eddy. J. Phys. Oceanogr., 47, 1737-1753, https://doi.org/10.1175/JPO-D-16-0266.1.

Arobone, E., and S. Sarkar, 2015: Effects of three-dimensionality on instability and turbulence in a frontal zone. J. Fluid Mech., 784, 252-273, https://doi.org/10.1017/jfm.2015.564.

Aubert, O., M. Le Bars, P. Le Gal, and P. S. Marcus, 2012: The universal aspect ratio of vortices in rotating stratified flows: Experiments and observations. J. Fluid Mech., 706, 34-45, https://doi.org/10.1017/jfm.2012.176.

Bachman, S. D., B. Fox-Kemper, J. R. Taylor, and L. N. Thomas, 2017: Parameterization of frontal symmetric instabilities. Part I: Theory for resolved fronts. Ocean Modell., 109, 72-95, https://doi.org/10.1016/j.ocemod.2016.12.003.

Baker, E. T., C. R. German, and H. Elderfield, 1995: Hydrothermal plumes over spreading-center axes: Global distributions and geological inferences. Seafloor Hydrothermal Systems: Physical, Chemical, Biological, and Geological Interactions, Geophys. Monogr., Vol. 91, Amer. Geophys. Union, 47-71, https://doi.org/10.1029/GM091p0047.

Bane, J. M., L. M. O'Keefe, and D. R. Watts, 1989: Mesoscale eddies and submesoscale, coherent vortices: Their existence near and interactions with the Gulf Stream. Mesoscale/ Synoptic Coherent Structures in Geophysical Turbulence, J. C. J. Nihoul and B. M. Jamart, Eds., Elsevier Oceanography Series, Vol. 50, Elsevier, 501-518, https://doi.org/10.1016/ S0422-9894(08)70204-6.

Billant, P., and F. Gallaire, 2005: Generalized Rayleigh criterion for non-axisymmetric centrifugal instabilities. J. Fluid Mech., 542, 365-379, https://doi.org/10.1017/S0022112005006464.

Boccaletti, G., R. Ferrari, and B. Fox-Kemper, 2007: Mixed layer instabilities and restratification. J. Phys. Oceanogr., 37, 2228 2250, https://doi.org/10.1175/JPO3101.1.

Bosse, A., and Coauthors, 2017: A submesoscale coherent vortex in the Ligurian Sea: From dynamical barriers to biological implications. J. Geophys. Res. Oceans, 122, 6196-6217, https:// doi.org/10.1002/2016JC012634.

Brannigan, L., H. Johnson, C. Lique, J. Nycander, and J. Nilsson, 2017: Generation of subsurface anticyclones at Arctic surface fronts due to a surface stress. J. Phys. Oceanogr., 47, 26532671, https://doi.org/10.1175/JPO-D-17-0022.1.
Buckingham, C. E., and Coauthors, 2016: Seasonality of submesoscale flows in the ocean surface boundary layer. Geophys. Res. Lett., 43, 2118-2126, https://doi.org/10.1002/ 2016GL068009.

—, N. Lucas, S. E. Belcher, T. Rippeth, A. Grant, J. Le Sommer, A. O. Ajayi, and A. Naveira Garabato, 2019: The contribution of surface and submesoscale processes to turbulence in the open ocean surface boundary layer. $J . A d v$. Model. Earth Syst., 11, 4066-4094, https://doi.org/10.1029/ 2019MS001801.

__ J. Gula, and X. Carton, 2021: The role of curvature in modifying frontal instabilities. Part I: Review of theory and presentation of a nondimensional instability criterion. J. Phys. Oceanogr., 51, 299-315, https://doi.org/10.1175/JPOD-19-0265.1.

Carton, X., and J. C. McWilliams, 1989: Barotropic and baroclinic instabilities of axisymmetric vortices in a quasigeostrophic model. Mesoscale/Synoptic Coherent Structures in Geophysical Turbulence, J. C. J. Nihoul and B. M. Jamart, Eds., Elsevier Oceanography Series, Vol. 50, Elsevier, 225-244, https://doi.org/ 10.1016/S0422-9894(08)70188-0.

Charney, J. G., 1947: The dynamics of long waves in a baroclinic westerly current. J. Meteor., 4, 136-162, https://doi.org/ 10.1175/1520-0469(1947)004<0136:TDOLWI>2.0.CO;2.

Chelton, D. B., M. G. Schlax, and R. M. Samelson, 2011: Global observations of nonlinear mesoscale eddies. Prog. Oceanogr., 91, 167-216, https://doi.org/10.1016/j.pocean.2011.01.002.

Cho, H.-R., T. G. Shepherd, and V. A. Vladimirov, 1993: Application of the direct Liapunov method to the problem of symmetric stability in the atmosphere. J. Atmos. Sci., 50, 822-836, https://doi.org/10.1175/1520-0469(1993)050<0822: AOTDLM $>2.0 . \mathrm{CO} ; 2$.

Colin de Verdière, A., 2012: The stability of short symmetric internal waves on sloping fronts: Beyond the traditional approximation. J. Phys. Oceanogr., 42, 459-475, https://doi.org/ 10.1175/JPO-D-11-067.1.

D'Asaro, E. A., 1988a: Generation of submesoscale vortices: A new mechanism. J. Geophys. Res., 93, 6685-6693, https:// doi.org/10.1029/JC093iC06p06685.

_- 1988b: Observations of small eddies in the Beaufort Sea. J. Geophys. Res., 93, 6669-6684, https://doi.org/10.1029/ JC093iC06p06669.

_ S. Walker, and E. Baker, 1994: Structure of two hydrothermal megaplumes. J. Geophys. Res., 99, 20361-20373, https:// doi.org/10.1029/94JC01846.

_ C. Lee, L. Rainville, R. Harcourt, and L. Thomas, 2011: Enhanced turbulence and energy dissipation at ocean fronts. Science, 332, 318-322, https://doi.org/10.1126/science.1201515.

de Marez, C., X. Carton, S. Corréard, P. L'Hégaret, and M. Morvan, 2020: Observations of a deep submesoscale cyclonic vortex in the Arabian Sea. Geophys. Res. Lett., 47, e2020GL087881, https://doi.org/10.1029/2020GL087881.

Deremble, B., 2016: Convective plumes in rotating systems. J. Fluid Mech., 799, 27-55, https://doi.org/10.1017/jfm.2016.348.

Eady, E. T., 1949: Long waves and cyclone waves. Tellus, 1A, 3352, https://doi.org/10.1111/j.2153-3490.1949.tb01265.x.

Ertel, H., 1942: Ein neuer hydrodynamischer wirbelsatz. Meteor. Z., 59, 271-281.

Fjortoft, R., 1950: Application of integral theorems in deriving criteria of stability for laminar flows and for the baroclinic circular vortex. Geophys. Publ., 17 (6), 1-52.

Flierl, G. R., 1988: On the instability of geostrophic vortices. J. Fluid Mech., 197, 349-388, https://doi.org/10.1017/S0022112088003283. 
Fox-Kemper, B., and Coauthors, 2011: Parameterization of mixed layer eddies. III: Implementation and impact in global ocean climate simulations. Ocean Modell., 39, 61-78, https://doi.org/ 10.1016/j.ocemod.2010.09.002.

Frenger, I., M. Münnich, N. Gruber, and R. Knutti, 2015: Southern Ocean eddy phenomenology. J. Geophys. Res. Oceans, 120, 7413-7449, https://doi.org/10.1002/2015JC011047.

Gallaire, F., and J. M. Chomaz, 2003: Three-dimensional instability of isolated vortices. Phys. Fluids, 15, 2113-2126, https:// doi.org/10.1063/1.1580481.

Garabato, A. C. N., and Coauthors, 2017: Vigorous lateral export of the meltwater outflow from beneath an Antarctic ice shelf. Nature, 542, 219-222, https://doi.org/10.1038/nature20825.

Gordon, A. L., E. Shroyer, and V. S. N. Murty, 2017: An intrathermocline eddy and a tropical cyclone in the Bay of Bengal. Sci. Rep., 7, 46218, https://doi.org/10.1038/srep46218.

Gula, J., M. J. Molemaker, and J. C. McWilliams, 2016: Topographic generation of submesoscale centrifugal instability and energy dissipation. Nat. Commun., 7, 12811, https:// doi.org/10.1038/ncomms12811.

— , T. M. Blacic, and R. E. Todd, 2019: Submesoscale coherent vortices in the Gulf Stream. Geophys. Res. Lett., 46, 2704 2714, https://doi.org/10.1029/2019GL081919.

Helfrich, K. R., and T. M. Battisti, 1991: Experiments on baroclinic vortex shedding from hydrothermal plumes. J. Geophys. Res., 96, 12 511-12 518, https://doi.org/10.1029/90JC02643.

Hogan, P. J., and H. E. Hurlburt, 2006: Why do intrathermocline eddies form in the Japan/East Sea? A modeling perspective. Oceanography, 19, 134-143, https://doi.org/10.5670/oceanog. 2006.50.

Holmes, R. M., L. N. Thomas, L. Thompson, and D. Darr, 2014: Potential vorticity dynamics of tropical instability vortices. J. Phys. Oceanogr., 44, 995-1011, https://doi.org/10.1175/JPOD-13-0157.1.

Holton, J. R., 1992: An Introduction to Dynamic Meteorology. 3rd ed. Academic Press, 511 pp.

Hoskins, B. J., 1974: The role of potential vorticity in symmetric stability and instability. Quart. J. Roy. Meteor. Soc., 100, 480482, https://doi.org/10.1002/qj.49710042520.

— models: Mathematical formulation and solution. J. Atmos. Sci., 29, 11-37, https://doi.org/10.1175/1520-0469(1972)029<0011: AFMMFA $>2.0 . \mathrm{CO} ; 2$.

— M. E. McIntyre, and A. W. Robertson, 1985: On the use and significance of isentropic potential vorticity maps. Quart. J. Roy. Meteor. Soc., 111, 877-946, https://doi.org/10.1002/ qj.49711147002.

Imberger, J., 1985: The diurnal mixed layer. Limnol. Oceanogr., 30, 737-770, https://doi.org/10.4319/lo.1985.30.4.0737.

Isern-Fontanet, J., J. Font, E. García-Ladona, M. Emelianov, C. Millot, and I. Taupier-Letage, 2004: Spatial structure of anticyclonic eddies in the Algerian basin (Mediterranean Sea) analyzed using the Okubo-Weiss parameter. Deep-Sea Res. II, 51, 3009-3028, https://doi.org/10.1016/j.dsr2.2004.09.013.

Jiao, Y., and W. K. Dewar, 2015: The energetics of centrifugal instability. J. Phys. Oceanogr., 45, 1554-1573, https://doi.org/ 10.1175/JPO-D-14-0064.1.

Kloosterziel, R. C., 2010: Viscous symmetric stability of circular flows. J. Fluid Mech., 652, 171-193, https://doi.org/10.1017/ S0022112009994149.

_ , and G. J. F. van Heijst, 1991: An experimental study of unstable barotropic vortices in a rotating fluid. J. Fluid Mech., 223, 1-24, https://doi.org/10.1017/S0022112091001301.
— , G. F. Carnevale, and P. Orlandi, 2007: Inertial instability in rotating and stratified fluids: Barotropic vortices. J. Fluid Mech., 583, 379-412, https://doi.org/10.1017/S0022112007006325.

,$- \ldots$, and -2017 : Equatorial inertial instability with full Coriolis force. J. Fluid Mech., 825, 69-108, https://doi.org/ 10.1017/jfm.2017.377.

Konstianoy, A. G., and I. M. Belkin, 1989: A survey of observations on intrathermocline eddies in the world ocean. Mesoscale/ Synoptic Coherent Structures in Geophysical Turbulence, J. C. J. Nihoul and B. M. Jamart, Eds., Elsevier Oceanography Series, Vol. 50, Elsevier, 821-841, https://doi.org/10.1016/ S0422-9894(08)70223-X.

Krishfield, R., J. Toole, A. Proshutinsky, and M.-L. Timmermans, 2008: Automated ice-tethered profilers for seawater observations under pack ice in all seasons. J. Atmos. Oceanic Technol., 25, 2091-2105, https://doi.org/10.1175/2008JTECHO587.1.

Kundu, P. K., and I. M. Cohen, 2008: Fluid Mechanics. 4th ed. Elsevier, 904 pp.

Lahaye, N., and V. Zeitlin, 2015: Centrifugal, barotropic and baroclinic instabilities of isolated ageostrophic anticyclones in the two-layer rotating shallow water model and their nonlinear saturation. J. Fluid Mech., 762, 5-34, https://doi.org/10.1017/ jfm.2014.631.

Lazar, A., A. Stegner, and E. Heifetz, 2013: Inertial instability of intense stratified anticyclones. Part I. Generalized stability criterion. J. Fluid Mech., 732, 457-484, https://doi.org/10.1017/ jfm.2013.412.

Legg, S., and J. C. McWilliams, 2001: Convective modifications of a geostrophic eddy field. J. Phys. Oceanogr., 31, 874-891, https://doi.org/10.1175/1520-0485(2001)031<0874:CMOAGE> 2.0.CO;2.

Li, Q., X. Mao, J. Huthnance, S. Cai, and S. Kelly, 2019: On internal waves propagating across a geostrophic front. J. Phys. Oceanogr., 49, 1229-1248, https://doi.org/10.1175/ JPO-D-18-0056.1.

Lilly, J. M., and P. B. Rhines, 2002: Coherent eddies in the Labrador Sea observed from a mooring. J. Phys. Oceanogr., 32, 585-598, https://doi.org/10.1175/1520-0485(2002)032<0585: CEITLS $>2.0 . \mathrm{CO} ; 2$.

Mahdinia, M., P. Hassanzadeh, P. S. Marcus, and C.-H. Jiang, 2017: Stability of three-dimensional Gaussian vortices in an unbounded, rotating, vertically stratified, Boussinesq flow: Linear analysis. J. Fluid Mech., 824, 97-134, https://doi.org/ 10.1017/jfm.2017.303.

Marchesiello, P., X. Capet, C. Menkes, and S. C. Kennan, 2011: Submesoscale dynamics in tropical instability waves. Ocean Modell., 39, 31-46, https://doi.org/10.1016/j.ocemod.2011. 04.011.

McDowell, S. E., and H. T. Rossby, 1978: Mediterranean water: An intense mesoscale eddy off the Bahamas. Science, 202, 10851087, https://doi.org/10.1126/science.202.4372.1085.

McWilliams, J. C., 1985: Submesoscale, coherent vortices in the ocean. Rev. Geophys., 23, 165-182, https://doi.org/10.1029/ RG023i002p00165.

- 2016: Submesoscale currents in the ocean. Proc. Roy. Soc. London, 472A, 1-32, https://doi.org/10.1098/rspa.2016.0117.

Meunier, T., and Coauthors, 2018: Intrathermocline eddies embedded within an anticyclonic vortex ring. Geophys. Res. Lett., 45, 7624-7633, https://doi.org/10.1029/2018GL077527.

Mooers, C. N. K., 1975: Several effects of baroclinic currents on the three-dimensional propagation of inertial-internal waves. Geophys. Fluid Dyn., 6, 277-284, https://doi.org/ 10.1080/03091927509365798. 
Naveira Garabato, A. C., and Coauthors, 2019: Rapid mixing and exchange of deep-ocean waters in an abyssal boundary current. Proc. Natl. Acad. Sci. USA, 116, 13 233-13 238, https:// doi.org/10.1073/pnas.1904087116.

Nurser, A. J. G., and S. Bacon, 2014: The Rossby radius in the Arctic Ocean. Ocean Sci., 10, 967-975, https://doi.org/10.5194/ os-10-967-2014.

Ooyama, K., 1966: On the stability of the baroclinic circular vortex: A sufficient criterion for instability. J. Atmos. Sci., 23, 43-53, https:// doi.org/10.1175/1520-0469(1966)023<0043:OTSOTB > 2.0.CO;2.

Paillet, J., B. Le Cann, X. Carton, Y. Morel, and A. Serpette, 2002: Dynamics and evolution of a northern meddy. J. Phys. Oceanogr., 32, 55-79, https://doi.org/10.1175/1520-0485(2002) $032<0055$ :DAEOAN $>2.0 . \mathrm{CO} ; 2$.

Penven, P., I. Halo, S. Pous, and L. Marié, 2014: Cyclogeostrophic balance in the Mozambique Channel. J. Geophys. Res. Oceans, 119, 1054-1067, https://doi.org/10.1002/2013JC009528.

Perfect, B., N. Kumar, and J. J. Riley, 2018: Vortex structures in the wake of an idealized seamount in rotating, stratified flow. Geophys. Res. Lett., 45, 9098-9105, https://doi.org/10.1029/ 2018 GL078703.

Rayleigh, L., 1917: On the dynamics of revolving fluids. Proc. Roy. Soc. London, 93, 148-154, https://doi.org/10.1098/ rspa.1917.0010.

Riser, S. C., W. B. Owens, H. T. Rossby, and C. C. Ebbesmeyer, 1986: The structure, dynamics, and origin of a small-scale lens of water in the western North Atlantic thermocline. J. Phys. Oceanogr., 16, 572-590, https://doi.org/10.1175/1520-0485(1986) $016<0572$ :TSDAOO $>2.0 . \mathrm{CO} ; 2$.

Roullet, G., and P. Klein, 2010: Cyclone-anticyclone asymmetry in geophysical turbulence. Phys. Rev. Lett., 104, 218501, https:// doi.org/10.1103/PhysRevLett.104.218501.

Rudnick, D. L., 2001: On the skewness of vorticity in the upper ocean. Geophys. Res. Lett., 28, 2045-2048, https://doi.org/ 10.1029/2000GL012265.

Shakespeare, C. J., 2016: Curved density fronts: Cyclogeostrophic adjustment and frontogenesis. J. Phys. Oceanogr., 46, 3193 3207, https://doi.org/10.1175/JPO-D-16-0137.1.

Shcherbina, A. Y., E. A. D'Asaro, C. M. Lee, J. M. Klymak, M. J. Molemaker, and J. C. McWilliams, 2013: Statistics of vertical vorticity, divergence, and strain in a developed submesoscale turbulence field. Geophys. Res. Lett., 40, 4706-4711, https:// doi.org/10.1002/grl.50919.

Skyllingstad, E. D., J. Duncombe, and R. M. Samelson, 2017: Baroclinic frontal instabilities and turbulent mixing in the surface boundary layer. Part II: Forced simulations. J. Phys. Oceanogr., 47, 2429-2454, https://doi.org/10.1175/JPO-D-160179.1 .

Smith, K. M., P. E. Hamlington, and B. Fox-Kemper, 2016: Effects of submesoscale turbulence on ocean tracers. J. Geophys. Res. Oceans, 121, 908-933, https://doi.org/10.1002/2015JC011089.

Solberg, H., 1936: Le mouvement d'inertie de l'atmosphere stable et son role dans la theorie des cyclones. Sixth Assembly, Paul Dupont, Edinburgh, Union Geodesique et Geophysique Internationale, 66-82.

Spall, M. A., 1995: Frontogenesis, subduction, and cross-front exchange at upper ocean fronts. J. Geophys. Res., 100, 2543 2557, https://doi.org/10.1029/94JC02860.

Speer, K. G., and J. Marshall, 1995: The growth of convective plumes at seafloor hot springs. J. Mar. Res., 53, 1025-1057, https://doi.org/10.1357/0022240953212972.
Stone, P. H., 1966: On non-geostrophic baroclinic stability. J. Atmos. Sci., 23, 390-400, https://doi.org/10.1175/15200469(1966)023<0390:ONGBS > 2.0.CO;2.

— 1970: On non-geostrophic baroclinic stability: Part II. J. Atmos. Sci., 27, 721-726, https://doi.org/10.1175/1520-0469(1970) $027<0721$ :ONGBSP $>2.0 . \mathrm{CO} ; 2$.

Taylor, J. R., and R. Ferrari, 2009: On the equilibration of a symmetrically unstable front via a secondary shear instability. J. Fluid Mech., 622, 103-113, https://doi.org/10.1017/ S0022112008005272.

$\longrightarrow$, and —, 2010: Buoyancy and wind-driven convection at mixed layer density fronts. J. Phys. Oceanogr., 40, 1222-1242, https://doi.org/10.1175/2010JPO4365.1.

Thomas, L. N., 2005: Destruction of potential vorticity by winds. J. Phys. Oceanogr., 35, 2457-2466, https://doi.org/10.1175/ JPO2830.1.

2008: Formation of intrathermocline eddies at ocean fronts by wind-driven destruction of potential vorticity. Dyn. Atmos. Oceans, 45, 252-273, https://doi.org/10.1016/j.dynatmoce. 2008.02.002.

, and J. R. Taylor, 2010: Reduction of the usable wind-work on the general circulation by forced symmetric instability. Geophys. Res. Lett., 37, L18606, https://doi.org/10.1029/ 2010GL044680.

- A. Tandon, and A. Mahadevan, 2008: Submesoscale processes and dynamics. Ocean Modeling in an Eddying Regime, Geophys. Monogr., Vol. 177, Amer. Geophys. Union, 17-38.

—_, J. R. Taylor, R. Ferrari, and T. M. Joyce, 2013: Symmetric instability in the Gulf Stream. Deep-Sea Res. II, 91, 91-110, https://doi.org/10.1016/j.dsr2.2013.02.025.

Thorpe, A. S., and R. Rotunno, 1989: Nonlinear aspects of symmetric instability. J. Atmos. Sci., 46, 1285-1299, https://doi.org/ 10.1175/1520-0469(1989)046<1285:NAOSI $>2.0 . C O ; 2$.

Timmermans, M.-L., J. Toole, A. Proshutinsky, R. Krishfield, and A. Plueddemann, 2008: Eddies in the Canada Basin, Arctic Ocean, observed from ice-tethered profilers. J. Phys. Oceanogr., 38, 133-145, https://doi.org/10.1175/2007JPO3782.1.

Toole, J. M., R. A. Krishfield, M.-L. Timmermans, and A. Proshutinsky, 2011: The ice-tethered profiler: Argo of the Arctic. Oceanography, 24, 126-135, https://doi.org/10.5670/oceanog.2011.64.

Wenegrat, J. O., J. Callies, and L. N. Thomas, 2018: Submesoscale baroclinic instability in the bottom boundary layer. J. Phys. Oceanogr., 48, 2571-2592, https://doi.org/10.1175/JPO-D-170264.1.

, L. N. Thomas, M. A. Sundermeyer, J. R. Taylor, E. A. D'Asaro, J. M. Klymak, R. K. Shearman, and C. M. Lee, 2020: Enhanced mixing across the gyre boundary at the Gulf Stream front. Proc. Natl. Acad. Sci. USA, 117, 17 607-17 614, https:// doi.org/10.1073/pnas.2005558117.

Yim, E., A. Stegner, and P. Billant, 2019: Stability criterion for the centrifugal instability of surface intensified anticyclones. J. Phys. Oceanogr., 49, 827-849, https://doi.org/10.1175/JPO-D-18-0088.1.

Zeitlin, V., 2018: Symmetric instability drastically changes upon inclusion of the full Coriolis force. Phys. Fluids, 30, 061701, https://doi.org/10.1063/1.5031099.

Zhang, Z., Y. Zhang, W. Wang, and R. X. Huang, 2013: Universal structure of mesoscale eddies in the ocean. Geophys. Res. Lett., 40, 3677-3681, https://doi.org/10.1002/grl.50736.

Zhao, M., M.-L. Timmermans, S. Cole, R. Krishfield, A. Proshutinsky, and J. Toole, 2014: Characterizing the eddy field in the Arctic Ocean halocline. J. Geophys. Res. Oceans, 119, 8800-8817, https:// doi.org/10.1002/2014JC010488. 